\title{
Diffeological Clifford algebras and pseudo-bundles of Clifford modules
}

\author{
Ekaterina Pervova
}

June 1,2021

\begin{abstract}
We consider the diffeological version of the Clifford algebra of a (diffeological) finite-dimensional vector space; we start by commenting on the notion of a diffeological algebra (which is the expected analogue of the usual one) and that of a diffeological module (also an expected counterpart of the usual notion). After considering the natural diffeology of the Clifford algebra, and considering which of its standard properties re-appear in the diffeological context (most of them), we turn to our main interest, which is constructing the pseudo-bundles of Clifford algebras associated to a given (finitedimensional) diffeological vector pseudo-bundle, and those of the usual Clifford modules (the exterior algebras). The substantial difference that emerges with respect to the standard context, and paves the way to various questions that do not have standard analogues, stems from the fact that the notion of a diffeological pseudo-bundle is very different from the usual bundle, and this under two main respects: it may have fibres of different dimensions, and even if it does not, its total and base spaces frequently are not smooth, or even topological, manifolds.

MSC (2010): 53C15, 15A69 (primary), 57R35, 57R45 (secondary).
\end{abstract}

\section{Introduction}

The concept of the diffeological space is due to J.M. Souriau [12, [13. The most comprehensive (and high-quality) source for diffeology is the excellent book [5], which provides also a fascinating account of how diffeology came about as a field. What might particularly be curious, given the context in which Clifford algebras usually appear, is the mentioning in the Preface of how diffeology takes sometimes a view in some sense complementary to that of the noncommutative geometry (a brief introduction to the latter field can be found, for instance, in [14]), and how it can be of help to whoever is not quite comfortable with heavy functional analysis or $C^{*}$-algebras 1

The content This paper is dedicated to considering Clifford algebras for (finite-dimensional) diffeological vector spaces and then the pseudo-bundles of such, along with pseudo-bundles of Clifford modules. The notion of a diffeological Clifford algebra does not bring much novelty with respect to the standard setting; it only needs to be noticed that, unless the algebra is built over a standard space - which is to say, $\mathbb{R}^{n}$ with the diffeology made up of all the usual smooth maps, - any smooth symmetric bilinear form is necessarily degenerate (see [5]), so there is not a unitary action on the corresponding exterior algebra (we consider some questions of which action does exist there).

On the other hand, interesting phenomena appear when we consider, instead of a single vector space, a collection of such that fibers over a diffeological space - in other words, a diffeological counterpart of a vector bundle. Such notion appeared originally in [4] (as a partial case of that of a diffeological fibre bundle; see also [5], Chapter 8); it was also treated in [15], where it is called a regular vector bundle, and employed in [1, where the term is a diffeological vector space over $X$. We opt for calling it a diffeological vector pseudo-bundle (although it is precisely the same thing), both because these objects, in general, are not bundles in the usual sense and because this avoids confusion with vector spaces proper.

A rough shape of such an object is a smooth surjective map $\pi: V \rightarrow X$, where $V$ and $X$ are diffeological spaces, and the pre-image of any point of $x$ is a finite-dimensional diffeological vector space.

\footnotetext{
${ }^{1}$ That would be myself, for instance.
} 
Given such $\pi: V \rightarrow X$, we endow it with a smooth symmetric bilinear form $g: X \rightarrow V^{*} \otimes V^{*}$ (as we have already commented, in general it cannot be a metric, meaning that it does not give a scalar product on individual fibres; we recall that it may not always have the maximal rank possible for a given fibre). We then turn to the subject of our main, which is pseudo-bundles of the corresponding Clifford algebras $C l(V, g)=\cup_{x \in X} C l\left(V_{x}, g(x)\right) \rightarrow X$ and those of (abstract) Clifford modules, where we concentrate on their behavior under the so-called gluing operation. Notice that all our vector spaces (in particular, the fibres $\left.\pi^{-1}(x) \subseteq V\right)$ are over real numbers.

The structure of the paper In order to make the paper self-contained, we collect in Section 1 all the main definitions and facts that are used therein, that is, diffeological spaces, diffeological vector spaces, Clifford algebras and modules, and diffeological algebras. In Section 2, which has somewhat expository nature (this material shall be at least implicitly known to anyone familiar with the diffeology field), we consider some instances of smooth actions of diffeological algebras on diffeological spaces. We comment on diffeological version of Clifford algebras and modules in Section 3. After recalling, in Section 4, the needed facts/constructions regarding diffeological vector pseudo-bundles and pseudo-metrics on them (this material is not new and comes from previous sources), in Sections 5 and 6 we consider the gluing operation for pseudo-bundles of Clifford algebras (Section 5) and those of Clifford modules (Section 6); the main result thus obtained is that, under appropriate compatibility conditions, the result of gluing is again a pseudo-bundle of Clifford algebras/modules in a natural way.

Acknowledgments The creation of this work is due to the influence of a non-mathematician colleague of mine, Prof. Riccardo Zucchi, whose good-naturedness, and the ability to provide subtle yet eloquent and inspiring comments and pointers, are beyond any praise. I also have a long-standing debt of gratitude to Prof. Paolo Piazza, to whom I owe my first true encounter with the Atiyah-Singer index theory, and in particular the first real encounter with Clifford algebras. Despite the referee reports on the next-to-last version of this paper being $O H$-polarized (for those who remember some chemistry), I am grateful to both of the authors of those reports. Finally, I am much grateful to the referees of the reports that came in after those, for many useful suggestions.

\section{Main definitions}

We collect here the standard definitions that are needed in what follows, with the exception of diffeological pseudo-bundles that are given a separate treatment in the section hereafter.

\subsection{Diffeological spaces and diffeologies}

We start by defining the main concepts regarding diffeological spaces proper that we need.

Diffeological spaces and smooth maps A diffeological space is just a set endowed with a diffeology, which is a sort of a substitute for the usual notion of a smooth structure; in and of itself, a diffeology is collection of maps into this set satisfying several properties. The precise definition is as follows.

Definition 1.1. ([13]) A diffeological space is a pair $\left(X, \mathcal{D}_{X}\right)$ where $X$ is a set and $\mathcal{D}_{X}$ is a specified collection of maps $U \rightarrow X$ (called plots) for each open set $U$ in $\mathbb{R}^{n}$ and for each $n \in \mathbb{N}$, such that for all open subsets $U \subseteq \mathbb{R}^{n}$ and $V \subseteq \mathbb{R}^{m}$ the following three conditions are satisfied:

1. (The covering condition) Every constant map $U \rightarrow X$ is a plot;

2. (The smooth compatibility condition) If $U \rightarrow X$ is a plot and $V \rightarrow U$ is a smooth map (in the usual sense) then the composition $V \rightarrow U \rightarrow X$ is also a plot;

3. (The sheaf condition) If $U=\cup_{i} U_{i}$ is an open cover and $U \rightarrow X$ is a set map such that each restriction $U_{i} \rightarrow X$ is a plot then the entire map $U \rightarrow X$ is a plot as well. 
Usually, instead of $\left(X, \mathcal{D}_{X}\right)$ one writes simply $X$ to denote a diffeological space. An easy example of a diffeological space is any smooth manifold, with the diffeology consisting of all usual smooth maps into that manifold.

Now, if we have two diffeological spaces, $X$ and $Y$, and a set map $f: X \rightarrow Y$ between them, then $f$ is called smooth if for every plot $p: U \rightarrow X$ of $X$ the composition $f \circ p$ is a plot of $Y$. The set of all smooth maps $X \rightarrow Y$ is denoted by $C^{\infty}(X, Y)$.

The underlying topology If $X$ is a diffeological space, there is a natural topology underlying its diffeological structure. It is called the $\mathbf{D}$-topology and can be characterized as follows: a subset $X^{\prime} \subset X$ is open in D-topology (and is said to be D-open) if and only if for every plot $p: \mathbb{R}^{m} \supset U \rightarrow X$ of $X$ the pre-image $p^{-1}\left(X^{\prime}\right)$ is a usual open set in $U \subset \mathbb{R}^{m}$. By Theorem 3.7 of [2] it actually suffices to ensure that this condition hold for the subset of plots defined on $\mathbb{R}$ (or its subintervals); the D-topology is defined by smooth curves only.

Typically (but not always), when considering some known topological space as a diffeological space, one would aim for a choice of diffeology on a known (topological) space such that the underlying Dtopology coincide with the existing topology. For that, it suffices to ensure that all plots be continuous in the usual sense. Notice that this applies only to specific situations (say, we wish to consider $\mathbb{R}$ as a diffeological space, while preserving its topology as a line); the set underlying the diffeological space is not required to carry any other structure.

Comparing diffeologies Given a set $X$, the set of all possible diffeologies on $X$ is partially ordered by inclusion (with respect to which it forms a complete lattice). More precisely, a diffeology $\mathcal{D}$ on $X$ is said to be finer than another diffeology $\mathcal{D}^{\prime}$ if $\mathcal{D} \subset \mathcal{D}^{\prime}$ (whereas $\mathcal{D}^{\prime}$ is said to be coarser than $\mathcal{D}$ ). Among all diffeologies, there is the finest one, which turns out to be the natural discrete diffeology and which consists of all locally constant maps $U \rightarrow X$; and there is also the coarsest one, which consists of all possible maps $U \rightarrow X$, for all $U \subseteq \mathbb{R}^{n}$ and for all $n \in \mathbb{N}$. It is called the coarse diffeology (or indiscrete diffeology by some authors).

Pushforwards and pullbacks For any diffeological space $X$, any set $X^{\prime}$, and any map $f: X \rightarrow X^{\prime}$ there exists a finest diffeology on $X^{\prime}$ that makes the map $f$ smooth. It is this diffeology that is called the pushforward of the diffeology of $X$ by the map $f$; the explicit description of this diffeology is as follows: $p^{\prime}: U \rightarrow X^{\prime}$ is a plot if and only if for any $u_{0} \in U$ there exists $u_{0} \in U^{\prime} \subseteq U$ such that $\left.p^{\prime}\right|_{U^{\prime}}=f \circ p$ for some plot $p$ of $X$. If, vice versa, we have a map $f: X^{\prime} \rightarrow X$ then $X^{\prime}$ can be endowed with the pullback diffeology, namely, the coarsest diffeology on $X$ such that $f$ is smooth; locally, its plots are precisely the maps $p: U \rightarrow X$ such that $f \circ p$ is a plot of $X^{\prime}$.

The subset diffeology Each subset $Y \subset X$ of a diffeological space $X$ carries a natural diffeology called subset diffeology (and so is always a diffeological space, in contrast with smooth manifolds, where most subsets are certainly not smooth (sub)manifolds). The subset diffeology is the coarsest diffeology such that the obvious inclusion map $Y \hookrightarrow X$ is smooth. From the practical point of view, it can be described as the set of all those plots of $X$ whose range is contained in $Y$.

The quotient diffeology Again, unlike smooth manifolds, the diffeological spaces always have other diffeological spaces as their quotients; these quotients are endowed a canonical diffeology, called the quotient diffeology. Specifically, if $X$ is a diffeological space and $\sim$ is an equivalence relation on $X$, then the quotient space $X / \sim$ is endowed with the diffeology that is the pushforward of the diffeology of $X$ by the natural projection $\pi: X \rightarrow X / \sim$. As is the case for all pushforward diffeologies, locally any plot $q$ of $X / \sim$ has form $q=\pi \circ p$ for some plot $p$ of $X$.

Disjoint unions and direct products Let $\left\{X_{i}\right\}_{i \in I}$ be a collection of diffeological spaces, where $I$ is a set of indices. The sum of $\left\{X_{i}\right\}_{i \in I}$ is defined as

$$
X=\coprod_{i \in I} X_{i}=\left\{(i, x) \mid i \in I \text { and } x \in X_{i}\right\} .
$$


The sum diffeology, or disjoint union diffeology ${ }^{2}$ on $X$ is the finest diffeology such that each natural injection $X_{i} \rightarrow \coprod_{i \in I} X_{i}$ is smooth; one property of the disjoint union diffeology is that any plot of it defined on a connected domain, is just a plot of one the components. The product diffeology $\mathcal{D}$ on the product $\prod_{i \in I} X_{i}$ is the coarsest diffeology such that for each index $i \in I$ the natural projection $\pi_{i}: \prod_{i \in I} X_{i} \rightarrow X_{i}$ is smooth. Locally every plot of the product diffeology is a collection of plots of the factors (for a finite product $X_{1} \times \ldots \times X_{n}$ the local shape of a plot is $\left(p_{1}, \ldots, p_{n}\right)$, where $p_{i}$ is a plot of $\left.X_{i}\right)$.

Functional diffeology Let $X, Y$ be two diffeological spaces, and let $C^{\infty}(X, Y)$ be the set of smooth maps from $X$ to $Y$. Let $\mathrm{EV}$ be the evaluation map, defined by

$$
\mathrm{EV}: C^{\infty}(X, Y) \times X \rightarrow Y \text { and } \mathrm{EV}(f, x)=f(x) .
$$

The functional diffeology on $C^{\infty}(X, Y)$ is the coarsest diffeology on it such that the evaluation map is smooth (although any diffeology such that EV is smooth may be called a functional diffeology).

\subsection{Diffeological vector spaces}

The next notion which we will (obviously) make use of is that of a diffeological vector space.

The definition of a diffeological vector space Let $V$ be a vector space over $\mathbb{R}$ (this is the only case that we consider here). A vector space diffeology on $V$ is any diffeology of $V$ such that the addition and the scalar multiplication are smooth, that is,

$$
[(u, v) \mapsto u+v] \in C^{\infty}(V \times V, V) \text { and }[(\lambda, v) \mapsto \lambda v] \in C^{\infty}(\mathbb{R} \times V, V),
$$

where $V \times V$ and $\mathbb{R} \times V$ are equipped with the product diffeology. A diffeological vector space is any vector space $V$ equipped with a vector space diffeology; all of our vector spaces will be finite-dimensional.

Subspaces and quotients Every vector space subspace $W$ of a diffeological vector space is itself a diffeological vector space for the subset diffeology; this is the diffeology with which it is canonically endowed. Likewise, any usual quotient space $V / W$ is a diffeological vector space for the quotient diffeology; this, again, is a canonical choice of a diffeology for it.

Linear maps Even in the finite-dimensional case (or maybe particularly so), in diffeology one needs to distinguish between linear maps and smooth linear maps. This is because, unless the diffeological vector space in question is a standard space, i.e., $\mathbb{R}^{n}$ with the standard diffeology, there will be at least one non-smooth linear map from it to some other diffeological vector space. We will see the implications of that when we come to the definition of the diffeological dual.

Direct sum of diffeological vector spaces Let $V_{1}, \ldots, V_{n}$ be diffeological vector spaces. Consider the usual direct sum $V=V_{1} \oplus \ldots \oplus V_{n}$ of this family; the space $V$, equipped with the product diffeology, is a diffeological vector space. The diffeology on the direct sum of an infinite family of vector spaces can be described as the coarsest diffeology such that such that the natural projection on each factor is a smooth linear map. Notice that the subset diffeology on each direct sum of a finite sub-family of factors is the same as described above.

Euclidean structures and pseudo-metrics The notion of a Euclidean diffeological vector space does not differ much from the usual notion of the Euclidean vector space. A diffeological space $V$ is Euclidean if it is endowed with a scalar product that is smooth with respect to the diffeology of $V$ and the standard diffeology of $\mathbb{R}$; that is, if there is a fixed map $\langle\rangle:, V \times V \rightarrow \mathbb{R}$ that has the usual properties of bilinearity, symmetricity, and definite-positiveness and that is smooth with respect to the diffeological

\footnotetext{
${ }^{2}$ We will mostly use the latter term, to avoid confusion with the vector space direct sum diffeology (see below), which is a product diffeology defined in the next sentence.
} 
product structure on $V \times V$ and the standard diffeology on $\mathbb{R}$. However, it is known (see, for instance, [5]) that a finite-dimensional diffeological vector space admits a smooth Euclidean structure if and only if it is diffeomorphic to the standard $\mathbb{R}^{n}$; in general, the maximal possible rank for a smooth symmetric bilinear form is equal to the dimension of its diffeological dual (see below). A form that achieves this rank always exists and is called a pseudo-metric.

Fine diffeology on vector spaces The fine diffeology on a vector space $V$ is the finest vector space diffeology on it; endowed with such, $V$ is called a fine vector space. In the finite-dimensional case (which is the only one we are treating here) any fine space is diffeomorphic to some $\mathbb{R}^{n}$, for appropriate $n$

The dual of a diffeological vector space The diffeological dual $V^{*}$ of a diffeological vector space $V$ (see [15], 17]) the set of smooth linear maps $V \rightarrow \mathbb{R}$ endowed with the functional diffeology, with respect to which it, itself, becomes a diffeological vector space. Note that if $V$ has finite dimension then this diffeology is standard, in the sense that $V^{*}$ is diffeomorphic to some $\mathbb{R}^{k}$ with the standard diffeology, for appropriate $k$ ([7]). This implies immediately (but is also easy to show directly) that unless a finite-dimensional $V$ is standard itself, its diffeological dual has strictly smaller dimension.

It should also be observed that there are many diffeological vector spaces, with not-so-large a diffeology, that have a trivial dual. It suffices to choose $n$ not-everywhere-smooth functions $f_{i}: \mathbb{R} \rightarrow \mathbb{R}$ and endow $\mathbb{R}^{n}$ with the vector space diffeology generated by the $n$ plots $\mathbb{R} \ni x \mapsto f_{i}(x) e_{i}$. Any smooth linear $\mathbb{R}$-valued map on this space must necessarily be trivial, yet the diffeology in question can be described rather concretely (it is essentially the extension of the ring of the usual smooth maps to $\mathbb{R}^{n}$ by the $n$ plots specified above).

The tensor product Let $V_{1}, \ldots, V_{n}$ be finite-dimensional diffeological vector spaces. Their usual tensor product is canonically endowed with the tensor product diffeology (see [15] and [17]), which is the pushforward of the product diffeology on the free product of vector spaces $V_{1} \times \ldots \times V_{n}$ by the universal map $V_{1} \times \ldots \times V_{n} \rightarrow V_{1} \otimes \ldots \otimes V_{n}$ (this is slightly different from the definitions in the above sources, but equivalent in the finite-dimensional case). The diffeological tensor product thus defined possesses the usual universal property ([15), Theorem 2.3.5): for any diffeological vector spaces $V_{1}, \ldots, V_{n}, W$ there is a diffeomorphism between the space $L^{\infty}\left(V_{1} \otimes \ldots \otimes V_{n}, W\right)$ of all smooth linear maps $V_{1} \otimes \ldots \otimes V_{n} \rightarrow W$ (endowed with the functional diffeolofy) and the space Mult ${ }^{\infty}\left(V_{1} \times \ldots \times V_{n}, W\right)$ of all smooth multilinear maps $V_{1} \times \ldots \times V_{n} \rightarrow W$, also endowed with the functional diffeology.

\subsection{Clifford algebras and Clifford modules}

In this section we recall briefly the standard notions of the Clifford algebra (see, for instance, [1]) for a real vector space equipped with a symmetric bilinear form, and then that of the Clifford module.

Clifford algebras There are a couple of ways to define a Clifford algebra, one more abstract and one more constructive; we recall both.

Definition 1.2. Let $V$ be a vector space equipped with a symmetric bilinear form $q($,$) . A Clifford$ algebra for $V$ is a unital algebra $C \ell(V, q)$ which is equipped with a map $\varphi: V \rightarrow C \ell(V, q)$ such that $\varphi(v)^{2}=-2 q(v, v) 1$, and which is universal among algebras equipped with such maps.

What the just-mentioned universality condition means precisely is the following: if $\varphi^{\prime}: V \rightarrow A$ is another map from $V$ to an algebra which satisfies $\varphi^{\prime}(v)^{2}=-2 q(v, v) 1$ then there is a unique algebra homomorphism $t: C \ell(V, q) \rightarrow A$ such that $\varphi^{\prime}=t \circ \varphi$.

\footnotetext{
${ }^{3}$ This is easy to see directly; assume that a given finite-dimensional fine space $V$ is already identified, as a vector space, with an appropriate $\mathbb{R}^{n}$. It suffices to see that the standard diffeology is the finest one on $\mathbb{R}^{n}$. Indeed, any diffeology contains all constant maps, so for any domain $U \subset \mathbb{R}^{m}$ (for whatever $m$ ) any diffeology contains the maps $U \ni u \mapsto e_{i}$, where $\left\{e_{i}\right\}_{i=1}^{n}$ is the canonical basis. Furthermore, any vector space diffeology contains all finite linear combinations with smooth functional coefficients of any collection of its plots. It follows that any usual smooth map $f: U \rightarrow \mathbb{R}^{n}$ is a plot for any vector space diffeology on $\mathbb{R}^{n}$, since we can write it as $u \mapsto \sum_{i=1}^{n} f_{i}(u) e_{i}$. Thus, the standard diffeology is indeed the finest vector space diffeology.
} 
An easy example is the exterior algebra of a given vector space, which corresponds to the bilinear form being identically zero.

The Clifford algebra can be equivalently defined in the following way.

Definition 1.3. Let $V$ be a vector space equipped with a symmetric bilinear form $q($, ). The Clifford algebra $C \ell(V, q)$ associated to $V$ and $q$ is the quotient of $T(V) / I(V)$ of the tensor algebra $T(V)=\sum_{r} V^{\otimes r}$ by the ideal $I(V) \subset T(V)$ generated by all the elements of the form $v \otimes w+w \otimes v+4 q(v, w)$, where $v, w \in V$.

The universal map is then the natural projection $T(V) \rightarrow C \ell(V, q)$.

Clifford modules Let $V$ and $q$ be as above.

Definition 1.4. A Clifford module is a vector space $E$ endowed with an action of the algebra $C \ell(V, q)$, that is, a unital algebra homomorphism $c: C \ell(V, q) \rightarrow \operatorname{End}(E)$.

If the space $E$ is Euclidean, i.e., endowed with a scalar product, one can speak of a unitary action, namely one for which $c(v)$ is an orthogonal transformation for each $v \in V$. Recall, as a main example, that the exterior algebra $\bigwedge^{*} V$ is a unitary Clifford module over $C \ell(V, q)$.

Grading and filtration on $C \ell(V, q)$ As is known, every Clifford algebra $C \ell(V, q)$ carries the following $\mathbb{Z}_{2}$ grading:

$$
C \ell(V, q)=C \ell(V, q)^{0} \oplus C \ell(V, q)^{1},
$$

where $C \ell(V, q)^{0}$ is the subspace generated by the products of an even number of elements of $V$, while $C \ell(V, q)^{1}$ is the subspace generated by the products of an odd number of elements of $V$; this is well-defined because $I(V)$ is generated by elements of even degree in $T(V)$.

Besides, $C \ell(V, q)$ inherits from $T(V)$ its filtration $T(V)=\sum_{k}\left(\sum_{r=0}^{k} V^{\otimes r}\right)$, via the natural projection. Therefore

$$
C \ell(V, q)=\sum_{k} C \ell^{k}(V, q), \text { where } C \ell^{k}(V, q)=\left\{v \in C \ell(V, q) \mid \exists u \in \sum_{r=0}^{k} V^{\otimes r} \text { such that }[u]=v\right\} .
$$

The use of natural projections allows also to define a surjective algebra homomorphism

$$
V^{\otimes k} \rightarrow C \ell^{k}(V, q) / C \ell^{k-1}(V, q) ;
$$

looking at its kernel, one sees that

$$
C \ell^{k}(V, q) / C \ell^{k-1}(V, q) \cong \Lambda^{k} V .
$$

This implies that the graded algebra associated to the just-described filtration on $C \ell(V, q)$, namely, the algebra $\oplus_{k} C \ell^{k}(V, q) / C \ell^{k-1}(V, q)$ is isomorphic to the external algebra $\wedge^{*} V$ (in particular, $\operatorname{dim}(C \ell(V, q))=$ $\left.2^{\operatorname{dim} V}\right)$.

\subsection{Diffeological algebras and diffeological modules}

The concepts of diffeological algebra and diffeological module are essentially obtained by adding the requirement of smoothness wherever it is (obviously) needed; this is at least implicit in the already existing works (see, for instance, [17]). We briefly recall them, occasionally making some of the reasoning explicit. Notice that an algebra for us is always an associative unital $\mathbb{R}$-algebra.

Diffeological algebras The definition of a diffeological algebra is of course an expected one: all operations are required to be smooth. We now state this formally.

Definition 1.5. Let $A$ be an algebra, and let $\mathcal{D}$ be a diffeology on the underlying set of $A$. The diffeology $\mathcal{D}$ is called a diffeological algebra structure on $A$, and $A$ is called a diffeological algebra, if the pair $(A, \mathcal{D})$ is a diffeological vector space with respect to the vector space structure of $A$ and, furthermore, the product map $A \times A \rightarrow A$ is smooth with respect to the product diffeology on $A \times A$. 
Generally speaking, a vector space admits many diffeologies that make it into a diffeological vector space (i.e., with respect to which the addition and the multiplication by scalar are smooth); it is the finest of these that is called the diffeological vector space structure. The same is a priori true for diffeological algebras, which leads us to the following definition:

Definition 1.6. Let $A$ be an algebra. The diffeological algebra structure on $A$ is the finest diffeology with respect to which $A$ is a diffeological vector space and the product in $A$ is smooth.

Note that a priori the diffeological algebra structure is finer than just a diffeological vector space structure, even the finest of the latter.

Subalgebras of a diffeological algebra Let $(A, \mathcal{D})$ be a diffeological algebra, and let $B \subset A$ be a subalgebra of $A$. As any subset of any diffeological space, $B$ carries a sub-diffeology coming from $\mathcal{D}$.

Lemma 1.7. The subalgebra $B$ endowed with the subset diffeology is a diffeological algebra.

Proof. Observe first of all that $B$, being a vector subspace of the diffeological vector space $A$, is a diffeological vector space for the subset diffeology (see [5], Section 3.5). Furthermore, if $p_{1}, p_{2}: U \rightarrow B$ are two plots of $B$ for the subset diffeology, that are defined on the same domain, then the map $p$ defined on $U$ and acting by $p(x)=p_{1}(x) p_{2}(x)$ is a plot of $A$, the latter being a diffeological algebra, and takes values in $B$, the set $B$ being a subalgebra of $A$. Therefore $p$ is a plot for the subset diffeology on $B$, which means that the product in $B$ is smooth for the subset diffeology, whence the conclusion.

Ideals of a diffeological algebra We now look at the ideals of a diffeological algebra $A$. Observe first of all that any ideal $I$, being a vector subspace of $A$, is a diffeological vector space with respect to the subset diffeology (as is the case for any vector subspace of any diffeological vector space, see [5], Section 3.5). The quotient $A / I$ is a diffeological vector space for the quotient diffeology (once again, as is the case for any diffeological vector space and any subspace of it, see [5], Section 3.6). Finally, $A / I$ is an algebra; the following easy lemma shows that it is a diffeological one.

Lemma 1.8. Let $A$ be a diffeological algebra, and let $I$ be an ideal of $A$. Then $A / I$ is a diffeological algebra for the quotient diffeology.

Proof. This follows from the definition of the quotient diffeology as the pushforward of the diffeology of $A$ by the natural projection; each operation in $A / I$ is the composition of the corresponding operation in $A$ with the natural projection, and therefore is smooth as (essentially) the composition of two smooth maps.

Diffeological module Let $A$ be a diffeological algebra, and let $E$ be a diffeological vector space.

Definition 1.9. We say that $E$ is a diffeological module over $A$ if there is a fixed homomorphism

$$
c: A \rightarrow L^{\infty}(E, E)
$$

that is smooth for the diffeology on $A$ and the functional diffeology on $L^{\infty}(E, E)$.

Note that, as has been observed in [6] and, I think, is implicit in [17, in general there is not a classical isomorphism $L^{\infty}(E, E) \cong E^{*} \otimes E$ (where $E^{*}$ is the diffeological dual of $E$, see [15], [17, and Section 2 above). Furthermore, while in the usual (finite-dimensional) vector space context $c$ can be seen as an element of $L(A, L(E, E)) \cong A^{*} \otimes L(E, E) \cong A^{*} \otimes E^{*} \otimes E$, this is not necessarily so in the diffeological context (on the other hand, as shown in [6], the usual construction does yield a smooth map $A^{*} \otimes L^{\infty}(E, E) \rightarrow L^{\infty}\left(\left(A^{*}\right)^{*}, L^{\infty}(E, E)\right)$, but it may not be an isomorphism, and $\left(A^{*}\right)^{*}$ may not be diffeomorphic to $A$ ).

\subsection{The exterior algebra of a diffeological vector space}

In this section we comment, not so much on the fact that the usual symmetrization and antisymmetrization operators (and as a consequence, the inner product and the exterior product) are smooth (this is expected), but on why they are so. 
Symmetrization and antisymmetrization operators Let $V$ be a diffeological vector space; let $\mathcal{S}_{n}(V)$ stand for the space of all symmetric $n$-tensors on $V$, and let $\mathcal{A}_{n}(V)$ be the space of all antisymmetric $n$-tensors on it. We use the usual normalized version of the the symmetrization operator:

$$
\mathrm{Sym}: \underbrace{V \otimes \ldots \otimes V}_{n} \ni v_{1} \otimes \ldots \otimes v_{n} \mapsto \frac{1}{n !} \sum_{\sigma \in S_{n}} v_{\sigma(1)} \otimes \ldots \otimes v_{\sigma(n)} \in \underbrace{V \otimes \ldots \otimes V}_{n},
$$

with $S_{n}$ standing for the group of permutations on $n$ elements and the operator being then extended by linearity.

This operator is smooth as a map $V_{1} \otimes \ldots \otimes V_{n} \rightarrow V_{1} \otimes \ldots \otimes V_{n}$, by the properties of a vector space diffeology and because its composition with the projection onto the component of the sum corresponding to any fixed $\sigma \in S_{n}$ is smooth. The smoothness of the latter follows easily from the definition of the tensor product diffeology as the pushforward of the relevant product diffeology (which is commutative in a tautological manner - in fact, its definition does not take the order of factors into account) and by writing each permutation as a composition of transpositions.

Lemma 1.10. The subset diffeology and the pushforward diffeology by Sym on the space $\mathcal{S}_{n}(V)$ of all symmetric $n$-tensors on $V$ coincide.

Proof. The pushforward diffeology on $\mathcal{S}_{n}(V)$ is by definition the finest such that Sym is smooth. On the other hand, this operator is smooth for the subset diffeology, being smooth as an operator into $V_{1} \otimes \ldots \otimes V_{n}$, and by the very definition of the subset diffeology. Thus, the pushforward diffeology is $a$ priori finer than the subset diffeology. The fact that they are actually the same comes from the fact that the restriction of Sym to $\mathcal{S}_{n}(V)$ is the identity. So if $p: U \rightarrow \mathcal{S}_{n}(V)$ is a plot for the subset diffeology, and $i: \mathcal{S}_{n}(V) \hookrightarrow V_{1} \otimes \ldots \otimes V_{n}$ is the natural inclusion, we have the equality $p=\operatorname{Sym} \circ(i \circ p)$; since $i \circ p$ is a plot of $V_{1} \otimes \ldots \otimes V_{n}$, we get that $p$ is a plot for the pushforward diffeology on $\mathcal{S}_{n}(V)$, and the statement is proven.

All the same reasoning holds for the usual antisymmetrization operator,

$$
\text { Alt }: \underbrace{V \otimes \ldots \otimes V}_{n} \ni v_{1} \otimes \ldots \otimes v_{n} \mapsto \frac{1}{n !} \sum_{\sigma \in S_{n}} \operatorname{sgn}(\sigma) v_{\sigma(1)} \otimes \ldots \otimes v_{\sigma(n)} \in \underbrace{V \otimes \ldots \otimes V}_{n} .
$$

Thus, this operator is smooth as well.

Lemma 1.11. The subset diffeology and the pushforward diffeology by Alt on the space $\mathcal{A}_{n}(V)$ of all antisymmetric $n$-tensors on $V$ coincide.

Proof. The proof is the same as in the case of the symmetrization operator, since in particular the idempotent property (i.e., Alt $\circ$ Alt $=$ Alt) holds for Alt just the same.

The exterior product Let $V$ again be a finite-dimensional diffeological space; as usual, the linear $n$-forms on $V$ (i.e., all antisymmetric covariant $n$-tensors) form the space

$$
\bigwedge^{n}(V):=\operatorname{Alt}(\underbrace{V^{*} \otimes \ldots \otimes V^{*}}_{n})
$$

which is also the space that previously we denoted by $\mathcal{A}_{n}\left(V^{*}\right)$. Let us consider the usual exterior product as a map

$$
\wedge: \bigwedge^{k}(V) \times \bigwedge^{l}(V) \rightarrow \bigwedge^{k+l}(V)
$$

between the diffeological vector spaces. Recall that this map acts by the normalized antisymmetrization of the tensor product of any two tensors, which immediately implies that it is smooth (note that the finite-dimensional case here coincides with the standard one, since the dual $V^{*}$ of a finite-dimensional diffeological $V$ always carries the standard diffeology). 


\section{Instances of diffeological algebras and diffeological modules}

We consider here several instances of diffeological algebras and diffeological modules, concentrating on providing examples of what changes when a well-known algebra is endowed with a diffeology.

\subsection{Algebras}

Our aim here is to consider some matrix algebras, endowed with various diffeologies, and answer a few obvious questions, such as: what are the diffeologies such that the matrix product is not smooth? or the trace? or the determinant? Surprisingly or not, it is quite easy to find matrix algebras and diffeologies where neither of these is smooth.

The trace This is probably the simplest case, meaning that it is easy to find examples of algebras (more precisely, put, on any algebra of square matrices, diffeologies) such that the trace is not a smooth function. A simple example of this sort readily comes by extending our examples of non-standard diffeological vector spaces.

Example 2.1. Let $V=\mathcal{M}_{2 \times 2}(\mathbb{R})$ be the usual algebra of $2 \times 2$ matrices with real coefficients; endow it with the vector space diffeology generated by the plot $p: \mathbb{R} \ni x \mapsto\left(\begin{array}{cc}0 & 0 \\ 0 & |x|\end{array}\right)$. Then it is obvious that the trace is not a smooth function (into the standard $\mathbb{R}$ ), since the composition trop, which is the map $x \mapsto|x|$, is not a smooth function.

The example just given is a rather crude attempt, since, as we will discover shortly, $V$ is not a diffeological algebra (it is easy to see that the matrix product is not smooth). Let us briefly see the case of the determinant.

The determinant It is also easy to see that the determinant is not a smooth function on $V$ of the above example. Indeed, since the diffeology in question is a vector space diffeology, the map $q: \mathbb{R} \ni x \mapsto$ $p(x)+\left(\begin{array}{ll}1 & 0 \\ 0 & 0\end{array}\right)$ is a plot of it (being the sum of $p$ with the constant plot $\left.x \mapsto\left(\begin{array}{ll}1 & 0 \\ 0 & 0\end{array}\right)\right)$. Once again, det $\circ q$ is the map $x \mapsto|x|$, so the determinant is not smooth as a map into the standard $\mathbb{R}$.

The product Finally, it is equally easy to see that the product map on $V$ is not a smooth map in $V$. It suffices to consider the product $\left(\begin{array}{ll}1 & 1 \\ 0 & 1\end{array}\right)\left(\begin{array}{cc}0 & 0 \\ 0 & |x|\end{array}\right)=\left(\begin{array}{cc}0 & |x| \\ 0 & |x|\end{array}\right)$. The matrix on the right is not a plot of $V$ (for any plot $p^{\prime}: U \rightarrow V$ of $V$ the $(1,2)$ th coefficient of $p(u)$ is an ordinary smooth function in $u$ ), so $V$ is not a diffeological algebra.

Generating an algebra diffeology Always in reference to our above example, the question of what is the analogue in case of algebras of the vector space diffeology generated by a given (collection of) $\operatorname{plot}(\mathrm{s})$, is rather natural. Of course, the abstract answer is obvious and is (at least) implicit elsewhere: if $A$ is an algebra and $\mathcal{A}$ is a collection of maps from domains of Euclidean spaces to $A$ then the algebra diffeology generated by $\mathcal{A}$ is the finest diffeology on $A$ that contains $\mathcal{A}$ and such that the addition, scalar multiplication, and the algebra product are all smooth. What we are wondering about is a concrete description, something which would give us an idea of, for instance, the algebra diffeology on the above $V$ generated by $p$.

Observation 2.2. The algebra diffeology on $V$ generated by $p$ is the vector space diffeology generated by the following four maps:

$$
\begin{aligned}
& p_{11}: \mathbb{R} \ni x \mapsto\left(\begin{array}{cc}
|x| & 0 \\
0 & 0
\end{array}\right), p_{12}: \mathbb{R} \ni x \mapsto\left(\begin{array}{cc}
0 & |x| \\
0 & 0
\end{array}\right), \\
& p_{21}: \mathbb{R} \ni x \mapsto\left(\begin{array}{cc}
0 & 0 \\
|x| & 0
\end{array}\right), p_{22}: \mathbb{R} \ni x \mapsto\left(\begin{array}{cc}
0 & 0 \\
0 & |x|
\end{array}\right) .
\end{aligned}
$$


Proof. We need to show two things: one, that the vector space diffeology generated by the maps $p_{i j}$ is an algebra diffeology, and, two, that any algebra diffeology on $V$ that contains $p=p_{22}$, also contains $p_{11}$, $p_{12}$, and $p_{21}$. Now, the reason why the vector space diffeology generated by these four maps is also an algebra diffeology is that any plot of it either is locally constant or locally filters through a plot of form

$$
\mathbb{R} \ni x \mapsto\left(\begin{array}{cc}
f_{11}(x)+g_{11}(x)\left|h_{11}(x)\right| & f_{12}(x)+g_{12}(x)\left|h_{12}(x)\right| \\
f_{21}(x)+g_{21}(x)\left|h_{21}(x)\right| & f_{22}(x)+g_{22}(x)\left|h_{22}(x)\right|
\end{array}\right),
$$

where $f_{i j}, g_{i j}, h_{i j}$ are some usual smooth functions. Now, since $|x|^{2}=x^{2}$ is a smooth function, the extension of the ring $C^{\infty}(\mathbb{R}, \mathbb{R})$ by the absolute value function $x \mapsto|x|$ is of degree 1 over $C^{\infty}(\mathbb{R}, \mathbb{R})$, which implies precisely that the product of two matrices as above is again a matrix of the same form, that is, the product is smooth as a map $V \times V \rightarrow V$.

Finally, in order to prove that an algebra diffeology on $V$ containing $p_{22}$, must also contain all the other maps $p_{i j}$, it suffices to consider the products

$$
\begin{gathered}
\left(\begin{array}{ll}
0 & 1 \\
0 & 0
\end{array}\right) \cdot\left(\begin{array}{cc}
0 & 0 \\
0 & |x|
\end{array}\right)=\left(\begin{array}{cc}
0 & |x| \\
0 & 0
\end{array}\right),\left(\begin{array}{cc}
0 & 0 \\
0 & |x|
\end{array}\right) \cdot\left(\begin{array}{cc}
0 & 0 \\
1 & 0
\end{array}\right)=\left(\begin{array}{cc}
0 & 0 \\
|x| & 0
\end{array}\right), \\
\left(\begin{array}{ll}
0 & 1 \\
0 & 0
\end{array}\right) \cdot\left(\begin{array}{cc}
0 & 0 \\
0 & |x|
\end{array}\right) \cdot\left(\begin{array}{ll}
0 & 0 \\
1 & 0
\end{array}\right)=\left(\begin{array}{cc}
|x| & 0 \\
0 & 0
\end{array}\right) .
\end{gathered}
$$

(The matrices on the right-hand side of these three expressions must be values at $x$ of some plot of the diffeological algebra $V$, and these values are respectively $p_{12}(x), p_{21}(x)$, and $p_{11}(x)$ ).

Are det and tr always smooth for a diffeological algebra? Suppose, on the other hand, that an algebra of matrices $\mathcal{M}_{n \times n}(\mathbb{R})$ is endowed with an algebra diffeology. It is then natural to wonder whether the determinant and the trace are necessarily smooth functions. The answer is negative and easily follows from the example already considered, that of $V=\mathcal{M}_{2 \times 2}(\mathbb{R})$ endowed with the algebra diffeology generated by the plot $p: x \mapsto\left(\begin{array}{cc}0 & 0 \\ 0 & |x|\end{array}\right)$.

Lemma 2.3. If $V$ is as above, det $: V \rightarrow \mathbb{R}$ and tr $: V \rightarrow \mathbb{R}$ are not smooth as functions into the standard $\mathbb{R}$.

Proof. The trace is not smooth since trop is the absolute value function, i.e., not a plot for the standard $\mathbb{R}$. As for the determinant, note first that, since any constant map is a plot for any diffeology, and the addition is smooth, the map $q: x \mapsto\left(\begin{array}{cc}1 & 0 \\ 0 & |x|\end{array}\right)$ is a plot of $V$. Since det $\circ q$ is again the absolute value function, it is not a plot of the standard $\mathbb{R}$, which implies that det is not a smooth function.

\subsection{Modules}

We now turn to illustrate in a concrete fashion the concept of a diffeological module. We first comment on the usual action of the matrix algebra $\mathcal{M}_{n \times n}(\mathbb{R})$ on a finite-dimensional diffeological vector space $V$, identified with $\mathbb{R}^{n}$ and endowed with some (non-standard) diffeology $\mathcal{D}_{V}$.

\subsubsection{The functional diffeology on $L^{\infty}(V, V)$ and multiplication by matrices}

Let us briefly explain the relation between the two. By the standard property of the functional diffeology, a map $t: U \rightarrow L^{\infty}(V, V)$ is a plot for the functional diffeology if (and only if) for every plot $q: U^{\prime} \rightarrow V$ of $V$ the evaluation of $t(u)$ on $s\left(u^{\prime}\right)$ is a plot of $V$, with the domain of definition $U \times U^{\prime}$. So if $\mathcal{M}_{n \times n}(\mathbb{R})$ is endowed with an algebra diffeology $\mathcal{D}_{\mathcal{M}}$, its action on $V$ by the usual left multiplication is smooth if and only if for every plot $A: U \rightarrow \mathcal{M}_{n \times n}(\mathbb{R})$ of the matrix algebra and for every plot $p: U^{\prime} \rightarrow V$ of $V$ the assignment

$$
U \times U^{\prime} \ni\left(u, u^{\prime}\right) \mapsto A(u) \cdot p\left(u^{\prime}\right) \in V
$$

defines again a plot of $V$. The condition, of course, remains the same if in place of $\mathcal{M}_{n \times n}(\mathbb{R})$ we consider any of its subalgebras (with an algebra diffeology). 


\subsubsection{The action of $\mathcal{M}_{2 \times 2}(\mathbb{R})$ on a non-standard $\mathbb{R}^{2}$}

This is one of the simplest examples of a diffeological module, which however indicates already the main differences of the concept upon introducing a diffeological structure.

The domain of action and the subalgebra with smooth action Let $V$ be a diffeological vector space, $V=\mathbb{R}^{2}$ with the vector space diffeology generated by the plot $p: x \mapsto(0,|x|)$, and let $\mathcal{A}=$ $\mathcal{M}_{2 \times 2}(\mathbb{R})$ the algebra of $2 \times 2$ matrices, whose diffeology we do not specify for the moment. Indeed, we are about to discover that $\mathcal{A}$ taken as a whole does not admit a smooth action on $V$, and this is because it contains matrices that while they obviously induce homomorphisms $V \rightarrow V$, these homomorphisms are not smooth. As an example, it suffices to take the matrix $\left(\begin{array}{ll}1 & 1 \\ 0 & 1\end{array}\right)$; in general, it is easy to observe that a matrix $A \in \mathcal{A}$ induces a smooth endomorphism of $V$ if and only if $(0,1)$ is an eigenvector of it. This means only the lower-triangular matrices (i.e., matrices $A$ such that $\left.(A)_{12}=0\right)$ act smoothly on $V$. Denote by $\mathcal{A}_{t r}$ the algebra of all such matrices.

The diffeology on the subalgebra acting Let us now consider the potential diffeologies on $\mathcal{A}_{t r}$. The ultimate condition that such diffeologies should satisfy is that the action-by-left-multiplication map

$$
c: \mathcal{A}_{t r} \rightarrow L^{\infty}(V, V)
$$

be smooth for the chosen diffeology on $\mathcal{A}_{t r}$ and the functional diffeology on $L^{\infty}(V, V)$; and this, by the characterization of functional diffeologies, means that, for every plot $q: U \rightarrow \mathcal{A}_{t r}$ of $\mathcal{A}_{t r}$ and for every plot $s: U^{\prime} \rightarrow V$ of $V$, the map $U \times U^{\prime} \ni\left(u, u^{\prime}\right) \mapsto q(u) \cdot s\left(u^{\prime}\right) \in V$ must be a plot of $V$.

Proposition 2.4. Let $\mathcal{A}_{t r}$ be endowed with a diffeology $\mathcal{D}_{\text {matr }}$ such that the left-multiplication action $c$ on $V$ is smooth. Then $\mathcal{D}_{\text {matr }}$ is the standard diffeology on $\mathcal{A}_{t r}$.

Proof. Let us consider a pair $(q, s)$, where $q: U \rightarrow \mathcal{A}_{t r}$ is a plot of $\mathcal{A}_{t r}$ and $s: U \rightarrow V$ is a plot $V$; let us see under which conditions the map $\left(u, u^{\prime}\right) \mapsto q(u) \cdot s\left(u^{\prime}\right)$ is a plot of $V$. Now, $s\left(u^{\prime}\right)=\left(s_{1}\left(u^{\prime}\right), s_{2}\left(u^{\prime}\right)\right)$, where $s_{1}: U^{\prime} \rightarrow \mathbb{R}$ is a usual smooth function, $s_{1} \in C^{\infty}\left(U^{\prime}, \mathbb{R}\right)$, while $s_{2}$ belongs to the extension of the ring $C^{\infty}\left(U^{\prime}, \mathbb{R}\right)$ by the absolute value function. In practice, this means that $s_{2}\left(u^{\prime}\right)=f\left(u^{\prime}\right)+g\left(u^{\prime}\right)\left|h\left(u^{\prime}\right)\right|$ for some $f, g, h \in C^{\infty}\left(U^{\prime}, \mathbb{R}\right)$. Note that the product $q(u) \cdot s\left(u^{\prime}\right)$ must again be of this form (with $U \times U^{\prime}$ in place of $\left.U^{\prime}\right)$.

Let us write $q(u)=\left(\begin{array}{cc}q_{11}(u) & 0 \\ q_{21}(u) & q_{22}(u)\end{array}\right)$; we need to show that the three functions $q_{i j}$ are ordinary smooth functions. Considering first the case of $s$ the constant plot with the value $(1,0)$ (and using the characterization of plots of $V$ just given), we immediately conclude that $q_{11}$ and $q_{21}$ must be ordinary smooth functions, while taking the constant plot with value $(0,1)$ we conclude that $q_{22}$ must have the form indicated for $s_{2}$, i.e., $q_{22}(u)=f_{1}(u)+g_{1}(u)\left|h_{1}(u)\right|$ for some $f_{1}, g_{1}, h_{1} \in C^{\infty}(U, \mathbb{R})$.

Suppose that $q_{22}$ is not a smooth function; then it has form $f_{1}(u)+g_{1}(u)\left|h_{1}(u)\right|$, where $g_{1}$ and $h_{1}$ are not everywhere zero. However, the diffeology in question is an algebra diffeology, so the following must also be a plot of it:

$$
u \mapsto\left(\begin{array}{cc}
q_{11}(u) & 0 \\
q_{21}(u) & q_{22}(u)
\end{array}\right) \cdot\left(\begin{array}{cc}
1 & 0 \\
1 & 1
\end{array}\right)=\left(\begin{array}{cc}
q_{11}(u) & 0 \\
q_{21}(u)+q_{22}(u) & q_{22}(u)
\end{array}\right) .
$$

Since we have already established that the $(2,1)$-th component of any plot of $\mathcal{A}_{t r}$ must be a smooth function, we obtain a contradiction with the assumption that $q_{22}$ is not smooth. The statement is proven.

A subalgebra with smooth action and non-standard diffeology There does however exist a subalgebra of $\mathcal{M}_{2 \times 2}(\mathbb{R})$ with smooth action on $V$ and non-standard diffeology. This is the subalgebra of diagonal matrices, endowed with the algebra diffeology generated by the plot $p(x)=\left(\begin{array}{cc}0 & 0 \\ 0 & |x|\end{array}\right)$ (this statement is quite obvious, so we make no further comment). 


\subsubsection{The case of $\mathcal{M}_{3 \times 3}(\mathbb{R})$ and non-standard $\mathbb{R}^{3}$}

Let us now consider a larger example. Specifically, we take $V=\mathbb{R}^{3}$ endowed with the vector space diffeology generated by the plot $p: \mathbb{R} \ni x \rightarrow|x| e_{3} \in V$; as in the case of dimension 2, consider $\mathcal{A}=\mathcal{M}_{3 \times 3}(\mathbb{R})$, the algebra of $(3 \times 3)$-matrices, that acts as usual, by left multiplication.

Smooth action on $V$ We can generalize a number of observations already made in the case of $n=2$, namely, a matrix $A \in \mathcal{A}$ defines a smooth endomorphism of $V$ if and only if $(0,0,1)$ is an eigenvector of it. Since this implies that $(A)_{13}=(A)_{23}=0$, we must restrict our attention to the subalgebra $\mathcal{A}^{\prime}$ of matrices satisfying this condition.

Diffeology on $\mathcal{A}^{\prime} \quad$ Let us consider the possible choice(s) of algebra diffeology on $\mathcal{A}^{\prime}$ that make its action on $V$ smooth. Let $p: U \ni u \mapsto\left(\begin{array}{ccc}p_{11}(u) & p_{12}(u) & 0 \\ p_{21}(u) & p_{22}(u) & 0 \\ p_{31}(u) & p_{32}(u) & p_{33}(u)\end{array}\right)$ be a plot of an algebra diffeology on $\mathcal{A}^{\prime}$. Taking products with the vector $(1,0,0)^{t}$ and $(0,1,0)^{t}$ allows us to see that $p_{11}, p_{12}, p_{21}, p_{22}: U \rightarrow \mathbb{R}$ must be ordinary smooth functions for the action to be smooth, while taking the product with $(0,0,1)^{t}$ shows that $p_{31}, p_{32}, p_{33}$ are of form $f(u)+g(u)|h(u)|$ for some smooth functions $f, g, h$. It remains to take into account the fact that the diffeology on $\mathcal{A}^{\prime}$ is an algebra diffeology, that is, that for any two plots $p, q$ of the form just indicated their product is again a plot. This amounts to checking that $(p(u) q(u))_{11}$, $(p(u) q(u))_{12},(p(u) q(u))_{21},(p(u) q(u))_{22}$ are in $C^{\infty}(U, \mathbb{R})$, while $(p(u) q(u))_{31},(p(u) q(u))_{32},(p(u) q(u))_{33}$ belong to the extension of $C^{\infty}(U, \mathbb{R})$ by the absolute value function; and this is established by the obvious calculation.

The block subalgebra $\mathcal{A}_{b l}$ and its action By $\mathcal{A}_{b l}$ we denote the subalgebra of $\mathcal{A}^{\prime}$ that satisfies the additional condition that $(A)_{31}=(A)_{32}=0$. It is quite easy to see that the coarsest algebra diffeology for which the action of $\mathcal{A}_{b l}$ on $V$ is smooth consists precisely of plots of form

$$
p: U \ni u \mapsto\left(\begin{array}{ccc}
p_{11}(u) & p_{12}(u) & 0 \\
p_{21}(u) & p_{22}(u) & 0 \\
0 & 0 & p_{33}(u)
\end{array}\right)
$$

where $p_{11}, p_{12}, p_{21}, p_{22}: U \rightarrow \mathbb{R}$ are ordinary smooth functions, while $p_{33}$ has form $u \mapsto f(u)+g(u)|h(u)|$, again for ordinary smooth functions $f, g, h$. Finally, it is also easy to see that the only other algebra diffeology on $\mathcal{A}_{b l}$ such that the left-multiplication map is smooth is the standard diffeology (more precisely, the subset diffeology relative to the standard diffeology on the standard algebra $\mathcal{M}_{3 \times 3}(\mathbb{R})$ ).

\subsubsection{Other considerations}

Given a finite-dimensional diffeological space $V$, there is the unique subspace $V_{0}$ of $V$ that is maximal for the following two properties:

1. the subset diffeology on $V_{0}$ is the standard diffeology;

2. there is another subspace $V_{1}$ of $V$ such that $V=V_{0} \oplus V_{1}$ as a diffeological vector space, that is, the diffeology of $V$ is the direct sum diffeology relative to the subset diffeology on $V_{0}$ and $V_{1}$.

The dimension of $V_{0}$ is that of the diffeological dual $V^{*}$ (see [7]).

Choose now a basis $\left\{v_{1}, \ldots, v_{k}\right\}$ of $V_{0}$ and a basis $\left\{v_{k+1}, \ldots, v_{n}\right\}$ of $V_{1}$. With respect to the basis $\left\{v_{1}, \ldots, v_{n}\right\}$ of $V$ thus obtained, a matrix $A \in \mathcal{M}_{n \times n}(\mathbb{R})$ defines a smooth endomorphism of $V$ only if we have:

$$
A=\left(\begin{array}{cc}
A_{k} & 0 \\
B & C
\end{array}\right)
$$

where $A_{k} \in \mathcal{M}_{k \times k}(\mathbb{R})$ and 0 is the zero $(k \times(n-k))$-matrix (then $B$ is of course of size $(n-k) \times k$ and $C$ is of size $(n-k) \times(n-k))$. Note that it is only a necessary condition, not a sufficient one. 
Denoting by $\mathcal{A}_{n, k}$ the algebra of all $(n \times n)$-matrices of this form (i.e., such that the upper right-hand block of size $k \times(n-k)$ is the zero matrix), we observe that (for the same reasons as it occurs in our examples) for any plot $p: U \rightarrow \mathcal{A}_{n, k}$ of an algebra diffeology on $\mathcal{A}_{n, k}$ for which the left-multiplication action on $V$ is smooth, the functions $p_{i j}: U \rightarrow \mathbb{R}$ given by taking the $(i, j)$-th coefficient of the matrix $p(u)$ are smooth in the usual sense for $i, j=1, \ldots, k$. Not much can be said in general about the rest of the functions $p_{i j}$, as it much depends on the specific diffeology of $V$ (more precisely, on its non-standard part).

\section{Diffeological Clifford algebras}

We now turn to the diffeological version of the Clifford algebra, associated to a finite-dimensional diffeological vector space.

\subsection{The definition of the diffeology}

The explicit construction of each Clifford algebra $C \ell(V, q)$ as a quotient of the tensor algebra $T(V)$ yields the obvious quotient diffeology on $C \ell(V, q)$ (which is the pushforward of the diffeology of $T(V)$ by the natural projection). This should be considered as the most natural choice for the diffeology on $C \ell(V, q)$ (the possible alternatives are rather artificial, so we do not discuss them).

The quotient diffeology from the tensor algebra Let us outline the construction of this diffeology. Recall first of all that each space $V^{\otimes k}$, with $k=1, \ldots, n=\operatorname{dim}(V)$, carries the tensor product diffeology (defined in [15] and [17, and recalled in Section 2). Now, the tensor algebra $T(V)$ is the direct sum of these spaces, $T(V)=\bigoplus_{k=1}^{n} V^{\otimes k}$; we endow it with the sum diffeology. Denote the resulting diffeology on $T(V)$ by $\mathcal{D}_{\otimes}$.

Lemma 3.1. The tensor algebra $T(V)$ considered with the diffeology $\mathcal{D}_{\otimes}$ is a diffeological algebra.

Proof. A direct sum of diffeological vector spaces is always a diffeological vector space for the product diffeology, so we only need to explain why the tensor product operation is smooth as a map

$$
V^{\otimes k} \times V^{\otimes l} \rightarrow T(V) .
$$

This follows automatically from the definition of the tensor product diffeology, which (for us) is the quotient diffeology corresponding to the product diffeology. Indeed, the product in $T(V)$ can be seen as the composition

$$
V^{\otimes k} \times V^{\otimes l} \rightarrow V^{\otimes(k+l)} \hookrightarrow T(V)
$$

of the quotient projection with the natural inclusion, both of which are smooth by construction 4

Since the Clifford algebra over $V$ is the quotient of $T(V)$ by its ideal, the quotient diffeology coming from $\mathcal{D}_{\otimes}$ makes $C \ell(V, q)$ into a diffeological algebra; we will consider $C \ell(V, q)$ with this diffeology.

The universal map for diffeological Clifford algebras The diffeological analogue of the universal property for Clifford algebras is the following statement.

Proposition 3.2. Let $V$ be a diffeological vector space endowed with a smooth bilinear symmetric form $q($,$) , let A$ be a diffeological algebra with unity, and let $f: V \rightarrow A$ be a smooth linear map such that

$$
f(v) \cdot f(w)+f(w) \cdot f(v)=-4 q(v, w) \cdot 1_{A} \text { for all } v, w \in V .
$$

Then there exists a (unique) smooth algebra homomorphism $\hat{f}: C \ell(V, q) \rightarrow A$ that extends $f$.

\footnotetext{
${ }^{4}$ Note that the natural embedding of each summand into a direct sum of vector spaces being smooth is a property of the product diffeology coming from vector space diffeologies. There is no analogue of it for a general product diffeology; then again, this is more because for a generic direct product there are no canonical inclusions of the factors.
} 
Proof. What we essentially need to do is to check whether the standard universal homomorphism $f^{\otimes}$ : $T(V) \rightarrow A$ extending $f$ is smooth. Indeed, by assumption on $f$, the homomorphism $f^{\otimes}$ yields a welldefined $C \ell(V, q) \rightarrow A$, which, by definition of the quotient diffeology, is smooth if and only if $f^{\otimes}$ is smooth. To conclude, observe that this latter property follows from the smoothness of the product in $A$; as an illustration, let us show, for instance, that the standard extension $\tilde{f}: V \otimes V \rightarrow A$ (given by $\tilde{f}(v \otimes w)=f(v) f(w))$ is smooth.

Indeed, let $p: U \rightarrow V \otimes V$ be a plot; recall that locally $p$ writes as $p=\pi \circ\left(p_{1}, p_{2}\right)$, where $p_{1}, p_{2}$ are plots of $V$ defined (up to passing to a suitably small sub-domain) on $U$ and $\pi: V \times V \rightarrow V \otimes V$ is the natural projection. Then we have $(\tilde{f} \circ p)(u)=p_{1}(u) p_{2}(u)$, which is locally a plot of $A$, since the multiplication in $A$ is smooth.

\subsection{The exterior algebra as a diffeological Clifford module}

It has been recalled above that for each Clifford algebra $C \ell(V, q)$ the exterior algebra $\wedge V$ is a Clifford module over $C \ell(V, q)$. We now show that this is true in the diffeological context as well; more precisely, we show that the standard action of $C \ell(V, q)$ on $\Lambda V$ is smooth. For the duration of this section we assume that $q$ is a (diffeological) scalar product.

The standard action $c$ The usual action $c: C \ell(V, q) \rightarrow \operatorname{End}(\bigwedge V)$ is obtained by first defining it on $V$ and then extending it to the rest of $C \ell(V, q)$; on $V$, this action is given by setting

$$
c(v)=\varepsilon(v)-i(v) \text { for all } v \in V,
$$

where $\varepsilon(v)$ acts on $\bigwedge V$ by the left exterior product, $\varepsilon(v)(\alpha)=v \wedge \alpha$, for all $\alpha \in \Lambda V$, and $i(v)$ is the adjoint of $\varepsilon(v)$ via $q$, i.e., it acts by

$$
i(v)\left(w_{1} \wedge \ldots \wedge w_{l}\right)=\sum_{i=1}^{l}(-1)^{i+1} w_{1} \wedge \ldots \wedge q\left(v, w_{i}\right) \wedge \ldots \wedge w_{l} .
$$

The action $c$ is smooth This essentially follows from the smoothness of all operations and that of $q$ (observe that, for instance, the smoothness of the exterior product is due to the diffeology of $\wedge V$ being the quotient diffeology of that of $T(V)$ and equivalently, the pushforward of the latter by the antisymmetrization operator).

The isomorphism $C \ell(V, q) \cong \bigwedge V$ Recall that (in the case when $q$ is a scalar product) there is a standard isomorphism $\sigma: C \ell(V, q) \rightarrow \bigwedge V$ acting by

$$
\sigma(x)=c(x)(1) \text { for every } x \in C \ell(V, q) .
$$

We have the following:

Proposition 3.3. The isomorphism $\sigma$ is smooth.

Proof. Let $p: U \rightarrow C \ell(V, q)$ be a plot of the Clifford algebra $C \ell(V, q)$; we need to show that $\sigma \circ p$ is a plot of $\bigwedge V$. As we have seen above, the action $c$ is smooth, which means that $c \circ p: U \rightarrow L^{\infty}(\bigwedge V, \bigwedge V)$ is a plot for the functional diffeology on $L^{\infty}(\bigwedge V, \bigwedge V)$. By 1.57 of [5], this is equivalent to the smoothness of the following map: $C: U \times \wedge V \rightarrow \wedge V$ acting by $C(u, \alpha)=c(p(u))(\alpha)$. The map $C$ being smooth means that its composition with any plot of $U \times \Lambda V$ is a plot of $\bigwedge V$. Observe now that the map $p^{\prime}: U \rightarrow U \times \bigwedge V$ given by $p^{\prime}(u)=(u, 1)$ is a plot of $U \times \wedge V$, and that $C \circ p^{\prime}=\sigma \circ p$, whence the conclusion.

\subsection{Smooth decompositions of diffeological vector spaces}

Before going further, we need to verify a few statements regarding some aspects of the behavior of diffeology with respect to pushforwards; we do so for diffeological vector spaces, this being a more general case. The following definition explains the matter. 
Definition 3.4. Let $V$ be a diffeological vector space, and let $V=V_{0} \oplus V_{1}$ be its decomposition into a direct sum of two subspaces. We say that this decomposition is smooth if the direct sum diffeology relative to the subset diffeologies on $V_{0}$ and $V_{1}$ coincides with the diffeology of $V$.

Note that a priori the direct sum diffeology is finer than $V$ 's own diffeology (that this can easily happen is illustrated by Example 2.5 in [7]). What we are now interested in (although we do not consider this point comprehensively), is what happens with (non)smoothness of a decomposition under linear maps.

Lemma 3.5. Let $V$ be a diffeological vector space, let $W$ be a vector space, and let $f: V \rightarrow W$ be a surjective linear map. Let $V^{\prime} \leqslant V$ be a subspace of $V$, and let $W^{\prime}=f\left(V^{\prime}\right)$ be the corresponding subspace of $W$. Endow $W$ with the pushforward of the diffeology of $V$ by the map $f$; then the corresponding subset diffeology on $W^{\prime}$ is in general coarser than the pushforward of the subset diffeology on $V^{\prime}$ by the map $\left.f\right|_{V^{\prime}}$. If $\operatorname{Ker}(f)$ splits off as a smooth direct summand then the two diffeologies coincide.

Proof. Let $\mathcal{D}_{s}$ be the subset diffeology on $W^{\prime}$, and let $\mathcal{D}_{p s h}$ be the pushforward diffeology on it. By its definition, $\mathcal{D}_{p s h}$ consists of plots of local form $\left.f\right|_{V^{\prime}} \circ p^{\prime}=f \circ p^{\prime}$, where $p^{\prime}$ is a plot for the subset diffeology on $V^{\prime}$, i.e. it is a plot of $V$ with range in $V^{\prime}$. Thus, $f \circ p^{\prime}$ is a plot for the pushforward diffeology on $W$, which by assumption takes values in $W^{\prime}$ only; therefore it is automatically a plot for the subset diffeology on $W$. We conclude that $\mathcal{D}_{p s h} \subseteq \mathcal{D}_{s}$.

Now let $q: U \rightarrow W^{\prime}$ be a plot of $\mathcal{D}_{s}$; this means that it is a plot of $W$ of taking values in $W^{\prime}$ only. And so it is a plot for the pushforward diffeology on $W$, i.e. that locally (on each open subset $U^{\prime} \subset U$ small enough) it has form $q=f \circ p$ for some plot $p: U^{\prime} \rightarrow V$. If the range of $p$ is contained in $V^{\prime}$ then $p$ is a plot for the subset diffeology on $V^{\prime}$, and we can conclude that $q$ is a plot for the pushforward of the subset diffeology on $V^{\prime}$ by $\left.f\right|_{V^{\prime}}$. So assume that the range of $p$ is not contained in $V^{\prime}$.

If $\operatorname{Ker}(f)$ splits off as a smooth direct summand, $V=V_{1} \oplus \operatorname{Ker}(f)$ then (on a small enough subset $\left.U^{\prime} \subset U\right)$ the plot $p$ can be represented as $p=p_{1} \oplus p_{k e r}$ where both $p_{1}$ and $p_{k e r}$ are plots of $V$, with ranges contained in $V_{1}$ and $\operatorname{Ker}(f)$ respectively. Observe furthermore that the equality $q=f \circ p$ implies that $p\left(U^{\prime}\right) \subset V^{\prime}+\operatorname{Ker}(f)$; and this implies, in turn, that the range of $p_{1}$ is actually contained in $V^{\prime}$. Thus, we actually have $q=f \circ p_{1}$, where $p_{1}$ is a plot for the subset diffeology on $V^{\prime}$, so $q$ is a plot of $\mathcal{D}_{p s h}$.

Remark 3.6. That the kernel of $f$ splits off as a smooth direct summand is a sufficient condition, but not a necessary one.

We now consider the implications of the above lemma on the behavior of smooth decompositions under projections (essentially, under taking quotients).

Lemma 3.7. Let $V$ be a diffeological vector space, let $W$ be a vector space, and let $f: V \rightarrow W$ be a surjective linear map; endow $W$ with the pushforward diffeology. Let $V=V_{0} \oplus V_{1}$ be a smooth decomposition of $V$ into a direct sum, and suppose that $f\left(V_{0}\right) \cap f\left(V_{1}\right)=\{0\}$. Then the decomposition $W=W_{0} \oplus W_{1}$, where $W_{i}=f\left(V_{i}\right)$ for $i=0,1$, is smooth.

Proof. The decomposition $V=V_{0} \oplus V_{1}$ being smooth means that every plot $p$ of $V$ locally has form $p_{0}+p_{1}$ where $p_{i}$ takes values in $V_{i}$ for $i=0,1$ (which is equivalent to saying that $p_{i}$ is a plot for the subset diffeology on $V_{i}$ ). Let us now consider an arbitrary plot $q$ of $W$. By definition of the pushforward diffeology, locally it has form $f \circ p$ for some plot $p$ of $V$ (to account for the constant plots, recall that $f$ is surjective). By linearity of $f$ and the above observation on the structure of plots of $V$, we obtain that $q=f \circ p_{0}+f \circ p_{1}$, where $q_{i}:=f \circ p_{i}$ takes values in $W_{i}$ for $i=0,1$ and, $p_{i}$ being a plot of $V$, is also a plot of $W$. The plot $q$ being arbitrary, we obtain that $W=W_{0} \oplus W_{1}$ is a smooth decomposition.

\subsection{The grading of a diffeological Clifford algebra}

Our aim here is to show that the standard grading of a diffeological Clifford algebra is in fact a smooth decomposition of it as a diffeological vector space. It obviously corresponds to the standard grading of a usual Clifford algebra:

$$
C \ell(V, q)=C \ell(V, q)^{0} \oplus C \ell(V, q)^{1} .
$$


Proposition 3.8. The grading of any diffeological Clifford algebra $C \ell(V, q)$ is a smooth direct sum decomposition.

Proof. What we have to show is that the direct sum (in the sense of vector spaces) diffeology on $C \ell(V, q)^{0} \oplus$ $C \ell(V, q)^{1}$ relative to the subset diffeologies on $C \ell(V, q)^{0}$ and $C \ell(V, q)^{1}$ coincides with the diffeology on $C \ell(V, q)$ (a priori the direct sum diffeology is finer). It suffices to show that each plot $p$ of $C \ell(V, q)$ locally writes as a sum $p=p_{0}+p_{1}$, where each $p_{i}$ is a plot of the subset diffeology on $C \ell(V, q)^{i}$ for $i=0,1$.

Recall that each plot of $C \ell(V, q)$ is locally a composition of a plot of $T(V)$ with its universal projection on $C \ell(V, q)$. Let $\pi: T(V) \rightarrow C \ell(V, q)$ be this projection, let $T^{0}(V)=\bigoplus_{r} V^{\otimes 2 r}$ be the subspace of tensors of even degree, and let $T^{1}(V)=\bigoplus_{r} V^{\otimes(2 r+1)}$ be the subspace of tensors of odd degree. This decomposition is smooth by the definition of the diffeology on $T(V)$. Furthermore, by the standard reasoning, $\pi\left(T^{0}(V)\right)=C \ell(V, q)^{0}$ and $\pi\left(T^{1}(V)\right)=C \ell(V, q)^{1}$. Then by Lemma 3.7, we obtain that $C \ell(V, q)=C \ell(V, q)^{0} \oplus C \ell(V, q)^{1}$ is a smooth decomposition for the pushforward of the diffeology of $T(V)$ by the map $\pi$, that is, the diffeology of $C \ell(V, q)$ as defined.

\subsection{The filtration of a diffeological Clifford algebra}

Let us now consider the standard filtration of $C \ell(V, q)$ by $C \ell^{k}(V, q) / C \ell^{k-1}(V, q)$, with $C \ell^{k}(V, q)$ defined, as usual, as the image of the restriction $\pi^{k}$ to $T^{k}(V):=\bigoplus_{r=0}^{k} V^{\otimes r}$ of the natural projection $\pi: T(V) \rightarrow$ $C \ell(V, q)$.

Lemma 3.9. The subset diffeology on $C \ell^{k}(V, q)$ relative to its inclusion $C \ell^{k}(V, q) \subset C \ell(V, q)$ coincides with the pushforward of the (subset) diffeology on $T^{k}(V) \subset T(V)$ by the map $\pi^{k}$.

Proof. This is a direct consequence of definitions involved. The subset diffeology on $C \ell^{k}(V, q)$ consists of all plots of $C \ell(V, q)$ with range contained in $C \ell^{k}(V, q)$. The diffeology of $C \ell(V, q)$ is the pushforward of the diffeology of $T(V)$ by the map $\pi$, so any plot of it has form $\pi \circ p$ for a plot $p$ of $T(V)$. By the definition of the map $\pi$, the range of $\pi \circ p$ is contained in $C \ell^{k}(V, q)$ if and only if the range of $p$ is contained in $T^{k}(V)$, on which we obviously have $\pi=\pi^{k}$, whence the conclusion.

This lemma has the following (expected) implication.

Proposition 3.10. The usual isomorphism $C \ell^{k}(V, q) / C \ell^{k-1}(V, q) \rightarrow \bigwedge^{k} V$ is a smooth map with smooth inverse (for the quotient diffeology on $C \ell^{k}(V, q) / C \ell^{k-1}(V, q)$ ).

\section{Diffeological vector pseudo-bundles and pseudo-metrics}

As we have just seen, an individual diffeological Clifford algebra, or Clifford module, represents simply a particular instance of the standard construction; what is more interesting is considering a collection of them, a diffeological counterpart of a vector bundle of Clifford algebras/modules. However, unless we are dealing with a standard case, it is frequently not a true bundle; instead, it is a diffeological counterpart of such, a more complicated object that was first introduced in [4] (see also [5]), and then considered, for example, in [15] and then in [1. The terminology was different in all these sources (it was just diffeological fibre bundle for [4, regular vector bundle in [15], and diffeological vector space over $X$ in [1]); we call it a diffeological vector pseudo-bundle, finding this term more convenient for stressing its difference from usual bundles (as well as avoiding confusion with individual vector spaces).

\subsection{Diffeological vector pseudo-bundles}

The main difference between a diffeological vector pseudo-bundle and a usual smooth vector bundle is the absence of local trivializations. Indeed, diffeological pseudo-bundles are frequently not locally trivial, or they are not bundles at all, in the sense that the fibre may easily not be the same over different points. An easy example of such is the wedge of a line and a plane, i.e., of $\mathbb{R}$ and $\mathbb{R}^{2}$, at the origin, which fibers over the wedge of two copies of $\mathbb{R}$ (one of which is the covering $\mathbb{R}$ and the other is the $x$-axis of the $\mathbb{R}^{2}$ ). The fibres are of two sorts; in one case, they are points (considered as trivial vector spaces, and in the other case they are lines of form $\{x\} \times \mathbb{R}$, with the obvious vector space structure on the second factor). 
It is easy to endow the two spaces, $\mathbb{R} \vee_{0} \mathbb{R}^{2}$ and $\mathbb{R} \vee_{0} \mathbb{R}$, with diffeologies such that the above-described projection $\mathbb{R} \vee_{0} \mathbb{R}^{2} \rightarrow \mathbb{R} \vee_{0} \mathbb{R}$ of one onto the other be smooth, and the subset diffeology on the pre-image of each point be a vector space diffeology. This means that we obtain what is called a diffeological vector pseudo-bundle, which below we formally define.

The formal definition Let $V$ and $X$ be two diffeological spaces, and let $\pi: V \rightarrow X$ be a smooth surjective map.

Definition 4.1. The map $\pi: V \rightarrow X$ is called a diffeological vector pseudo-bundle, if for every $x \in X$ the pre-image $\pi^{-1}(x)$ is endowed with the structure of a vector space such that the following three conditions are satisfied:

1. the map $V \times_{X} V \rightarrow V$ induced by the addition within each fibre is smooth for the subset diffeology on $V \times_{X} V$ relative to its inclusion $V \times_{X} V \subset V \times V$ and the product diffeology on the latter;

2. the map $\mathbb{R} \times V \rightarrow V$ induced by the fibrewise scalar multiplication is smooth for the product diffeology on $\mathbb{R} \times V$ relative to the standard diffeology on $\mathbb{R}$;

3. the zero section is smooth as a map $X \rightarrow V$.

We will also use the term pseudo-bundle to refer to just the total space $V$. As already mentioned, the requirement of the existence of an atlas of local trivializations is absent; in particular, having fibres of different dimensions is explicitly admitted. An example of this has already been described in the beginning of this section, and we add some more below. Other instances appear in the literature, also arising from independent contexts, such as the internal tangent bundle to the union of coordinate axes in $\mathbb{R}^{2}$ constructed in [1].

Operations on vector pseudo-bundles The usual operations on smooth vector bundles (direct sum, tensor product, and so on) can still be defined for diffeological vector pseudo-bundles, although due to the absence of local trivializations this is done with a different approach. It appeared first in [15], Chapter 5 (some details are added in [8]).

- Sub-bundles and quotient pseudo-bundles. Let $\pi: V \rightarrow X$ be a finite-dimensional diffeological vector pseudo-bundle, and let $W \subset V$ be such that for every $x \in X$ the intersection $W \cap \pi^{-1}(x)$ is a vector subspace of $\pi^{-1}(x)$. Then $\left.\pi\right|_{W}: W \rightarrow X$, where $W$ is endowed with the subset diffeology relative to $V$, is also a diffeological vector pseudo-bundle, called a sub-bundle of $V$ (in general, it is also a pseudo-bundle). Vice versa, any collection

$$
\bigcup_{x \in X} W_{x} \text { such that } W_{x} \leqslant \pi^{-1}(x)
$$

of vector subspaces, one for each fibre, is a sub-bundle of $V$ for the subset diffeology and the restriction of $\pi$ onto it.

Let now $W \subset V$ be a sub-bundle of $\pi: V \rightarrow X$. Then $W$ defines an obvious equivalence relation on $V$, by taking the quotient of each fibre $\pi^{-1}(x)$ over the subspace $W \cap \pi^{-1}(x)$. The quotient space $V / W$,

$$
V / W=\bigcup_{x \in X}\left(\pi^{-1}(x) /\left(W \cap \pi^{-1}(x)\right)\right),
$$

endowed with the quotient diffeology and the obvious projection onto $X$, becomes a vector pseudobundle itself and is called the quotient pseudo-bundle. Obviously, the subset diffeology on each $\pi^{-1}(x) /\left(W \cap \pi^{-1}(x)\right) \subset V / W$ coincides with its quotient diffeology relative the subset diffeology on $\pi^{-1}(x)$.

- Direct sum of two pseudo-bundles. Let $\pi_{1}: V_{1} \rightarrow X$ and $\pi_{2}: V_{2} \rightarrow X$ be two diffeological vector pseudo-bundles with the same base space. Their direct sum, as a set, is

$$
V_{1} \oplus V_{2}:=V_{1} \times_{X} V_{2}=\left\{\left(v_{1}, v_{2}\right) \mid \pi_{1}\left(v_{1}\right)=\pi_{2}\left(v_{2}\right), v_{i} \in V_{i}, i=1,2\right\} \subset V_{1} \times V_{2} ;
$$


it is endowed with the obvious factorwise operations of addition and scalar multiplication and with the subset diffeology relative to the product diffeology on $V_{1} \times V_{2}$, as well as with the obvious projection $\pi_{1} \oplus \pi_{2}$ onto $X$. It is a matter of technicality then (see, for instance, [15]) that $\pi_{1} \oplus \pi_{2}$ : $V_{1} \oplus V_{2} \rightarrow X$ is a diffeological vector pseudo-bundle with the fibre

$$
\left(\pi_{1} \oplus \pi_{2}\right)^{-1}(x)=\pi_{1}^{-1}(x) \oplus \pi_{2}^{-1}(x)
$$

(as diffeological vector spaces) for every $x \in X$.

- Tensor product of two pseudo-bundles. Let, again, $\pi_{1}: V_{1} \rightarrow X$ and $\pi_{2}: V_{2} \rightarrow X$ be two diffeological vector pseudo-bundles. Their tensor product is defined as the quotient pseudo-bundle of the direct product product bundle $V_{1} \times_{X} V_{2}$ over its sub-bundle $W$ which is defined by requiring each fibre $W_{x}$ of $W$ to be precisely the kernel of the universal projection $\pi_{1}^{-1}(x) \times \pi_{2}^{-1}(x) \rightarrow$ $\pi_{1}^{-1}(x) \otimes \pi_{2}^{-1}(x)$. By construction, each fibre of this quotient has form $\pi_{1}^{-1}(x) \otimes \pi_{2}^{-1}(x)$.

- The dual pseudo-bundle. Let $\pi: V \rightarrow X$ be a diffeological vector pseudo-bundle, and let

$$
V^{*}=\cup_{x \in X}\left(\pi^{-1}(x)\right)^{*}
$$

be the union of the diffeological duals of all its fibres. Each dual obtains its diffeological vector space structure as the dual of the diffeological vector space $\pi^{-1}(x) \subset V$. The set $V^{*}$ is then endowed with the obvious projection onto $X$ and with the smallest diffeology relative to which all fibres recover their original diffeology as the subset diffeology.

That such a diffeology exists, and the following characterization of its plots of the dual bundle diffeology, comes from [15], Definition 5.3.1 and Proposition 5.3.2. Let $U$ be a domain of some $\mathbb{R}^{l}$. A map $p: U \rightarrow V^{*}$ is a plot for the dual bundle diffeology on $V^{*}$ if and only if for every plot $q: U^{\prime} \rightarrow V$ the map

$$
Y^{\prime} \ni\left(u, u^{\prime}\right) \mapsto p(u)\left(q\left(u^{\prime}\right)\right) \text { on } Y^{\prime}=\left\{\left(u, u^{\prime}\right) \mid \pi^{*}(p(u))=\pi\left(q\left(u^{\prime}\right)\right) \in X\right\} \subset U \times U^{\prime}
$$

is smooth for the subset diffeology of $Y^{\prime} \subset \mathbb{R}^{l+\operatorname{dim}\left(U^{\prime}\right)}$ and the standard diffeology of $\mathbb{R}$.

- The pseudo-bundle of linear maps. Let $\pi_{1}: V_{1} \rightarrow X$ and $\pi_{2}: V_{2} \rightarrow X$ be two finite-dimensional diffeological vector pseudo-bundles over the same base space $X$. For each $x \in X$ consider the space $L^{\infty}\left(\left(V_{1}\right)_{x},\left(V_{2}\right)_{x}\right)$ of smooth linear maps $\left(V_{1}\right)_{x} \rightarrow\left(V_{2}\right)_{x}$ endowed with the functional diffeology; this is a (finite-dimensional) diffeological vector space. The union

$$
\mathcal{L}\left(V_{1}, V_{2}\right):=\bigcup_{x \in X} L^{\infty}\left(\left(V_{1}\right)_{x},\left(V_{2}\right)_{x}\right)
$$

endowed with the obvious projection $\pi_{L}$ onto $X$, is a diffeological vector pseudo-bundle, that carries the following diffeology, called the pseudo-bundle functional diffeology. It is the smallest diffeology generated by all the maps $p: U \rightarrow \mathcal{L}\left(V_{1}, V_{2}\right)$, with $U$ being a domain of some $\mathbb{R}^{m}$, that possess the following property: for every plot $q: \mathbb{R}^{m^{\prime}} \supset U^{\prime} \rightarrow V_{1}$ the map

$$
\left(u, u^{\prime}\right) \mapsto p(u)\left(q\left(u^{\prime}\right)\right) \text { defined on } Y^{\prime}=\left\{\left(u, u^{\prime}\right) \mid \pi_{L}(p(u))=\pi_{1}\left(q\left(u^{\prime}\right)\right)\right\} \subset U \times U^{\prime}
$$

(and taking values in $V_{2}$ ) is smooth for the subset diffeology of $Y^{\prime} \subset \mathbb{R}^{m+m^{\prime}}$ and the diffeology of $V_{2}$.

Pseudo-bundle diffeology generated by some plots Let $X$ be a diffeological space, and let $\pi$ : $V \rightarrow X$ be a surjective map, where $V$ is a set such that for every $x \in X$ the pre-image $\pi^{-1}(x)$ carries a vector space structure.

Definition 4.2. Let $\mathcal{A}=\left\{p_{i}: U_{i} \rightarrow V \mid i \in I\right\}$ be a collection of maps such that $\pi \circ p_{i}$ is a plot of $X$ for all $i \in I$. The pseudo-bundle diffeology on $V$ generated by $\mathcal{A}$ is the smallest diffeology on $V$ that contains $\mathcal{A}$ and with respect to which $\pi: V \rightarrow X$ becomes a diffeological vector pseudo-bundle.

The existence of such a diffeology can be easily inferred from Proposition 4.16 of [1. Note that the notion of the pseudo-bundle diffeology generated by a set of given plots is distinct from that of the generated diffeology proper and that of the vector space diffeology generated by a plot. 
Examples Obviously, usual vector bundles satisfy the definition of a diffeological vector pseudo-bundle (as is formally established in [9, they actually serve as instances of a kind of diffeological assembly, called gluing, which is described in the next section). Other simple examples include all projections $\mathbb{R}^{k} \times \mathbb{R}^{m} \rightarrow \mathbb{R}^{k}$, where $\mathbb{R}^{k}$ is endowed with any diffeology, $\mathbb{R}^{m}$ with any vector space diffeology, and the total space, with the product diffeology. We will also give an example of a non-trivial non-manifold pseudobundle when it comes to discussing the so-called pseudo-metrics (substitutes of Riemannian metrics) on diffeological vector pseudo-bundles.

\subsection{More complicated pseudo-bundles: diffeological gluing}

Diffeological gluing can be thought of as a partial way to compensate for the absence of local trivializations in the concept of a diffeological vector pseudo-bundles, although it is not aimed to describe all possible pseudo-bundles (and likely does not). The idea is, just like many usual smooth vector bundles can be seen as the result of identification, along the transition functions, of several copies of the product bundle $\mathbb{R}^{n+k} \rightarrow \mathbb{R}^{n}$, so many diffeological pseudo-bundles can be obtained as the result of diffeological gluing of several trivial (or almost trivial, although this case we will avoid) pseudo-bundles of form $\mathbb{R}^{n_{i}+k_{i}} \rightarrow \mathbb{R}^{n_{i}}$. There are two main differences: one is that these trivial pseudo-bundles may have varying dimensions (and/or diffeologies), and the gluing may not be along diffeomorphisms, nor is it required, for the subsets being identified, to have non-empty interior. Still, the latter case is a generalization of the former (the rigorous statement and the proof can be found in [9]).

\subsubsection{Gluing of diffeological spaces and of diffeological vector pseudo-bundles}

Here we define formally the operation of gluing and the resulting diffeology, first for individual diffeological spaces and then for diffeological vector pseudo-bundles (the latter actually amounts to the former performed twice). The main aspect is that of the choice of diffeology, since from the topological/set-theoretic point of view this construction is well-known.

The gluing of two diffeological spaces Underlying this operation is the standard definition of gluing of two topological spaces. Although this can be defined in a more general context, we limit ourselves to the case of gluing along maps that are invertible (the assumption that they are smooth is always present). Then, as is standard, if $X_{1}, X_{2}$ are two topological spaces and $f: X_{1} \supset Y_{1} \rightarrow Y_{2} \subset X_{2}$ is a homeomorphism then the result of gluing $X_{1}$ and $X_{2}$ along $f$ is the space $X=\left(X_{1} \sqcup X_{2}\right) / \sim$, where $\sim$ is the equivalence relation given by $x_{1} \sim x_{1}$ for $x_{1} \in X_{1} \backslash Y_{1}, x_{2} \sim x_{2}$ for $x_{2} \in X_{2} \backslash Y_{2}$, and $Y_{1} \ni y_{1}=f\left(y_{1}\right) \in Y_{2}$. We will denote the space $X$ thus obtained by $X_{1} \cup_{f} X_{2}$ :

$$
X_{1} \cup_{f} X_{2}:=\left(X_{1} \sqcup X_{2}\right) / \sim .
$$

Definition 4.3. Let $X_{1}$ and $X_{2}$ be diffeological spaces, and let $f: X_{1} \supseteq Y_{1} \rightarrow Y_{2} \subseteq X_{2}$ be a map smooth for the subset diffeologies on $Y_{1}$ and $Y_{2}$. The gluing diffeology on the space $X_{1} \cup_{f} X_{2}$ is the quotient diffeology corresponding to the disjoint union diffeology on $X_{1} \sqcup X_{2}$; endowed with this diffeology, $X_{1} \cup_{f} X_{2}$ is said to be the result of diffeological gluing of $X_{1}$ to $X_{2}$ along $f$.

The gluing of two diffeological vector pseudo-bundles This is a direct sequence of the previous construction, under appropriate assumptions on the gluing maps. Specifically, let

$$
\pi_{1}: V_{1} \rightarrow X_{1} \text { and } \pi_{2}: V_{2} \rightarrow X_{2}
$$

be two (finite-dimensional) diffeological vector pseudo-bundles, let

$$
f: X_{1} \supset Y_{1} \rightarrow Y_{2} \subset X_{2}
$$

be a diffeomorphism for the subset diffeologies on $Y_{1}$ and $Y_{2}$, and let

$$
\tilde{f}: \pi_{1}^{-1}\left(Y_{1}\right) \rightarrow \pi_{2}^{-1}\left(Y_{2}\right)
$$


be a smooth map that is linear on each fibre and is a lift of $f$, namely, $\pi_{1} \circ f=\tilde{f} \circ \pi_{2}$. This latter condition ensures that between the diffeological spaces $V_{1} \cup_{\tilde{f}} V_{2}$ and $X_{1} \cup_{f} X_{2}$ there is a well-defined (smooth) map

$$
\pi_{1} \cup_{(\tilde{f}, f)} \pi_{2}: V_{1} \cup_{\tilde{f}} V_{2} \rightarrow X_{1} \cup_{f} X_{2}
$$

induced by $\pi_{1}$ and $\pi_{2}$, and it is easy to check (see [8]) that $\pi_{1} \cup_{(\tilde{f}, f)} \pi_{2}$ is again a diffeological vector pseudo-bundle.

\subsubsection{Gluing and other operations}

Recall that in the case of the usual smooth vector bundles, the operations on them are defined via their restrictions to the charts of a given atlas of local trivializations. What happens in the diffeological context is a procedure that in some sense is an inverse of this standard strategy: the operations are defined on the whole pseudo-bundles, and then one wonders what, if any, relation there is with our diffeological substitute for such atlas, which is a representation as the result of gluing of some finite number of trivial diffeological pseudo-bundles.

What we find, under this perspective is that in a number of cases the two commute. In particular, if we are also given a pseudo-bundle endowed with a so-called pseudo-metric (see the next section), this allows us to reduce the constructions of the corresponding pseudo-bundle of Clifford algebras and that of the exterior algebras (seen as the pseudo-bundle of Clifford modules; see the last section), essentially to the case of standard trivial bundles, or at least diffeologically trivial bundles.

The commutativity problem The question of whether the gluing operation on pseudo-bundles commutes with the usual operations on vector bundles (such as the direct sum, tensor product, and taking the dual bundle) is therefore a very natural one. Below we summarize the answer to it (which is respectively yes, yes, no for the three main operations just listed, see [8] and [9] for more details).

To fix the notation, consider two pairs of pseudo-bundles with their respective gluings. One pair is $\pi_{1}: V_{1} \rightarrow X_{1}$ and $\pi_{2}: V_{2} \rightarrow X_{2}$ that are glued along the maps $f: X_{1} \supset Y_{1} \rightarrow Y_{2} \subset X_{2}$ (a diffeomorphism for the subset diffeologies on $Y_{1}$ and $\left.Y_{2}\right)$ and $\tilde{f}: \pi_{1}^{-1}\left(Y_{1}\right) \rightarrow \pi_{2}^{-1}\left(Y_{2}\right)$ (smooth and linear on each fibre), yielding the pseudo-bundle

$$
\pi_{1} \cup_{(\tilde{f}, f)} \pi_{2}: V_{1} \cup_{\tilde{f}} V_{2} \rightarrow X_{1} \cup_{f} X_{2} .
$$

Let the other pair be $\pi_{1}^{\prime}: V_{1}^{\prime} \rightarrow X_{1}$ and $\pi_{2}^{\prime}: V_{2}^{\prime} \rightarrow X_{2}$; these are glued along the same map $f$ on the bases and a given lift $\tilde{f}^{\prime}:\left(\pi_{1}^{\prime}\right)^{-1}\left(Y_{1}\right) \rightarrow\left(\pi_{2}^{\prime}\right)^{-1}\left(Y_{2}\right)$ of it, yielding the pseudo-bundle

$$
\pi_{1}^{\prime} \cup_{(\tilde{f}, f)} \pi_{2}^{\prime}: V_{1}^{\prime} \cup_{\tilde{f}}, V_{2}^{\prime} \rightarrow X_{1} \cup_{f} X_{2} .
$$

The comparisons to make would be between the following pseudo-bundles.

Direct sum Consider the following direct sum pseudo-bundles:

$$
\pi_{1} \oplus \pi_{1}^{\prime}: V_{1} \oplus V_{1}^{\prime} \rightarrow X_{1} \text { and } \pi_{2} \oplus \pi_{2}^{\prime}: V_{2} \oplus V_{2}^{\prime} \rightarrow X_{2} .
$$

These can be glued along the maps $f$ and its lift

$$
\tilde{f} \oplus \tilde{f}^{\prime}: \pi_{1}^{-1}\left(Y_{1}\right) \oplus\left(\pi_{1}^{\prime}\right)^{-1}\left(Y_{1}\right) \rightarrow \pi_{2}^{-1}\left(Y_{2}\right) \oplus\left(\pi_{2}^{\prime}\right)^{-1}\left(Y_{2}\right) .
$$

It turns out that the result of this gluing, the pseudo-bundle

$$
\left(\pi_{1} \oplus \pi_{1}^{\prime}\right) \cup_{\left(\tilde{f} \oplus \tilde{f}^{\prime}, f\right)}\left(\pi_{2} \oplus \pi_{2}^{\prime}\right):\left(V_{1} \oplus V_{1}^{\prime}\right) \cup_{\tilde{f} \oplus \tilde{f}^{\prime}}\left(V_{2} \oplus V_{2}^{\prime}\right) \rightarrow X_{1} \cup_{f} X_{2},
$$

is canonically diffeomorphic, with diffeomorphism that is a lift of the identity diffeomorphism of the bases, to the pseudo-bundle

$$
\left(\pi_{1} \cup_{(\tilde{f}, f)} \pi_{2}\right) \oplus\left(\pi_{1}^{\prime} \cup_{\left(\tilde{f}^{\prime}, f\right)} \pi_{2}^{\prime}\right):\left(V_{1} \cup_{\tilde{f}} V_{2}\right) \oplus\left(V_{1}^{\prime} \cup_{\tilde{f}^{\prime}} V_{2}^{\prime}\right) \rightarrow X_{1} \cup_{f} X_{2} .
$$

In other words, gluing commutes with the direct sum. 
Tensor product The analogous question for the tensor product takes the following form. We state right away that between the two possible (pre-gluing) tensor products $V_{1} \otimes V_{1}^{\prime}$ and $V_{2} \otimes V_{2}^{\prime}$ there is the naturally (fibrewise) defined map

$$
\tilde{f} \otimes \tilde{f}^{\prime}: \pi_{1}^{-1}\left(Y_{1}\right) \otimes\left(\pi_{1}^{\prime}\right)^{-1}\left(Y_{1}\right) \rightarrow \pi_{2}^{-1}\left(Y_{2}\right) \otimes\left(\pi_{2}^{\prime}\right)^{-1}\left(Y_{2}\right),
$$

which is smooth, linear on each fibre, and a lift of $f$. This allows to form the glued pseudo-bundle

$$
\left(\pi_{1} \otimes \pi_{1}^{\prime}\right) \cup_{\left(\tilde{f} \otimes \tilde{f}^{\prime}, f\right)}\left(\pi_{2} \otimes \pi_{2}^{\prime}\right):\left(V_{1} \otimes V_{1}^{\prime}\right) \cup_{\tilde{f} \otimes \tilde{f}^{\prime}}\left(V_{2} \otimes V_{2}^{\prime}\right) \rightarrow X_{1} \cup_{f} X_{2} .
$$

The question is whether it is diffeomorphic to the tensor product pseudo-bundle

$$
\left(\pi_{1} \cup_{(\tilde{f}, f)} \pi_{2}\right) \otimes\left(\pi_{1}^{\prime} \cup_{\left(\tilde{f}^{\prime}, f\right)} \pi_{2}^{\prime}\right):\left(V_{1} \cup_{\tilde{f}} V_{2}\right) \otimes\left(V_{1}^{\prime} \cup_{\tilde{f}^{\prime}} V_{2}^{\prime}\right) \rightarrow X_{1} \cup_{f} X_{2} .
$$

Also in this case the answer is positive.

Dual pseudo-bundles Assume that $f$ is smoothly invertible. We now wish to compare the following two pseudo-bundles:

$$
\left(\pi_{1} \cup_{(\tilde{f}, f)} \pi_{2}\right)^{*}:\left(V_{1} \cup_{\tilde{f}} V_{2}\right)^{*} \rightarrow X_{1} \cup_{f} X_{2} \text { and } \pi_{2}^{*} \cup_{\left(\tilde{f}^{*}, f^{-1}\right)} \pi_{1}^{*}: V_{2}^{*} \cup_{\tilde{f}^{*}} V_{1}^{*} \rightarrow X_{2} \cup_{f^{-1}} X_{1} .
$$

In general, they are not diffeomorphic: since over the domain of gluing the resulting fibre is always the target space of the lifted part of the pair of maps that define the gluing, we must also have, for each $y_{1} \in Y_{1}$ (equivalently, for each $y_{2} \in Y_{2}$ ) that $\left(\pi_{1}^{-1}\left(y_{1}\right)\right)^{*}$ is diffeomorphic, as a diffeological vector space, to $\left(\pi_{2}^{-1}\left(f\left(y_{1}\right)\right)\right)^{*}$.

Note also that, even if the equality $\left(\pi_{1}^{-1}\left(y_{1}\right)\right)^{*}=\left(\pi_{2}^{-1}\left(f\left(y_{1}\right)\right)\right)^{*}$ holds for all $y \in Y$, it is not sufficient to ensure that $\left(\pi_{1} \cup_{(\tilde{f}, f)} \pi_{2}\right)^{*}=\pi_{2}^{*} \cup_{\left(\tilde{f}^{*}, f^{-1}\right)} \pi_{1}^{*}$. It suffices to think of the open annulus and the open Möbius strip, both considered with their usual smooth structure and their usual fibering over a circle. These smooth structures are a particular case of a diffeological structure, of course, and the projection onto the circle (also with its standard diffeology) is a particular case of a diffeological vector pseudo-bundle for each of them. Obviously, these two spaces are not even homeomorphic, let alone diffeomorphic.

Thus, another necessary condition for the gluing-dual commutativity is that the dual map $\tilde{f}^{*}$ be a diffeomorphism of its domain with its image. It is shown in [10] that this condition becomes sufficient under the additional assumption that the factors of gluing admit the so-called compatible pseudo-metrics (see the next section).

\subsection{Pseudo-metrics on pseudo-bundles}

It is known (see [5]) that a finite-dimensional diffeological vector space in general does not admit a scalar product, therefore the straightforward extension of the notion of the Riemannian metric to diffeological vector pseudo-bundles does not make much sense. Indeed, such an extension would be a smooth section $g: X \rightarrow V^{*} \otimes V^{*}$ of a given finite-dimensional diffeological vector pseudo-bundle $\pi: V \rightarrow X$ with each value $g(x)$ a smooth scalar product on the fibre; thus, on most pseudo-bundles it would not exist at all. It is therefore replaced by the notion of a pseudo-metric on a pseudo-bundle, which is obtained by replacing scalar products on fibres with symmetric bilinear forms of maximal rank. Such an object does not always exists either, but it is applicable to a much wider class of pseudo-bundles.

\subsubsection{What is a pseudo-metric on a pseudo-bundle?}

We now describe an obvious extension of the notion of a pseudo-metric from the case of a finite-dimensional diffeological vector space to that of a diffeological vector pseudo-bundle.

The notion Let $\pi: V \rightarrow X$ be a diffeological vector pseudo-bundle.

Definition 4.4. A pseudo-metric on the pseudo-bundle $\pi: V \rightarrow X$ is any smooth section $g$ of the pseudo-bundle $\pi^{*} \otimes \pi^{*}: V^{*} \otimes V^{*} \rightarrow X$ such that for every $x \in X$ the form $g(x)$ is a pseudo-metric on the diffeological vector space $\pi^{-1}(x)$, that is, it is semi-definite positive symmetric of rank $\operatorname{dim}\left(\left(\pi^{-1}(x)\right)^{*}\right)$.

Note that by Theorem 2.3.5 of [15] $g(x)$ is indeed a bilinear map on $\pi^{-1}(x)$, so the definition is well-posed. We have already announced that it does not, however, always exist (we give an example below). 
Pseudo-bundles that do not admit pseudo-metrics For the definition as stated, it is quite easy to find examples of pseudo-bundles that do not admit any pseudo-metric. Namely, what happens (as in the case of the example below) is, there is a smooth section $X \rightarrow V^{*} \otimes V^{*}$ that satisfies all the conditions to be a pseudo-metric except one: it does not have the maximal rank possible everywhere, but only outside of some positive codimension subset. (It is quite similar to an example that appears in [8]).

Example 4.5. Let $V=\mathbb{R}^{2}$ and $X=\mathbb{R}$; let $\pi: V \rightarrow X$ be the projection on the $x$-axis, $\pi(x, y)=x$. Endow $X$ with the standard diffeology, and endow $V$ with the pseudo-bundle diffeology generated by the plot $p: \mathbb{R}^{2} \rightarrow V$ defined by $p(u, v)=(u, u|v|)$.

The following was proven in [9. The main point is that the rank of the prospective pseudo-metric on $V$ would have to be zero everywhere except over the origin, where it should be 1; despite the diffeology being non-standard, it is impossible to find a smooth section satisfying this condition.

Lemma 4.6. If $\pi: V \rightarrow X$ is the pseudo-bundle of Example 4.5, it does not admit a pseudo-metric.

The basic reason for this is that the evaluation of any prospective pseudo-metric on a constant non-zero section is proportional to the $\delta$-function, with non-zero coefficient.

\subsubsection{Pseudo-bundles obtained by gluing and pseudo-metrics}

We have already indicated the pseudo-bundles obtained by gluing of some (a finite number of) standard (diffeologically trivial) bundles as our main object of interest 5 Since our final goal is to consider the corresponding pseudo-bundles of Clifford algebras (and then those of Clifford modules), we need to clarify how pseudo-metrics behave with respect to the gluing; indeed, this behavior is not uniquely defined.

The compatibility condition This condition is in fact immediately obvious (out of geometric considerations) as a necessary one for the existence of some kind of induced pseudo-metric. Let $\pi_{1}: V_{1} \rightarrow X_{1}$ and $\pi_{2}: V_{2} \rightarrow X_{2}$ be two pseudo-bundles, with a gluing between them, given by a smooth invertible $f: X_{1} \supset Y \rightarrow f(Y) \subset X_{2}$ and its smooth fibrewise linear lift $\tilde{f}: \pi_{1}^{-1}(Y) \rightarrow \pi_{2}^{-1}(f(Y))$. Suppose that each pseudo-bundle $V_{i}$ carries a pseudo-metric $g_{i}$.

Definition 4.7. We say that $g_{1}$ and $g_{2}$ are compatible if for every $y \in Y$ and for all $v_{1}, v_{2} \in \pi_{1}^{-1}(y)$ we have

$$
g_{1}(y)\left(v_{1}, v_{2}\right)=g_{2}(f(y))\left(\tilde{f}\left(v_{1}\right), \tilde{f}\left(v_{2}\right)\right) .
$$

Inductions into glued spaces Note (see [9]) that the following obvious inclusions

$$
\begin{gathered}
i_{1}: X_{1} \backslash Y \hookrightarrow X_{1} \cup_{f} X_{2}, \quad i_{2}: X_{2} \hookrightarrow X_{1} \cup_{f} X_{2}, \\
j_{1}: V_{1} \backslash \pi_{1}^{-1}(Y) \hookrightarrow V_{1} \cup_{f} V_{2}, \quad j_{2}: V_{2} \hookrightarrow V_{1} \cup_{f} V_{2}
\end{gathered}
$$

are all inductions 6 Furthermore, the images of $i_{1}$ and $i_{2}$ are disjoint and cover $X_{1} \cup_{f} X_{2}$ (the same holds for $j_{1}, j_{2}$, and $V_{1} \cup_{\tilde{f}} V_{2}$ ). We will frequently make use of this fact and the notation (and the respective counterparts of these in other cases) when defining various maps involving glued pseudo-bundles/spaces.

The induced pseudo-metric The following statement, proven in [9], shows that a pseudo-bundle obtained by gluing carries a natural pseudo-metric, as long as the factors of gluing are endowed with compatible ones.

Theorem 4.8. Let $\pi_{1}: V_{1} \rightarrow X_{1}$ and $\pi_{2}: V_{2} \rightarrow X_{2}$ be two finite-dimensional diffeological vector pseudobundles, let $(\tilde{f}, f)$ be a gluing between them, and let $g_{1}$ and $g_{2}$ be pseudo-metrics on $V_{1}$ and, respectively, $V_{2}$ compatible with respect to the gluing. Define $\tilde{g}: X_{1} \cup_{f} X_{2} \rightarrow\left(V_{1} \cup_{\tilde{f}} V_{2}\right)^{*} \otimes\left(V_{1} \cup_{\tilde{f}} V_{2}\right)^{*}$ as

$$
\tilde{g}(x)= \begin{cases}\left(\left(j_{1}^{-1}\right)^{*} \otimes\left(j_{1}^{-1}\right)^{*}\right) \circ g_{1}\left(i_{1}^{-1}(x)\right) & \text { for } x \in i_{1}\left(X_{1} \backslash Y\right) \\ \left(\left(j_{2}^{-1}\right)^{*} \otimes\left(j_{2}^{-1}\right)^{*}\right) \circ g_{2}\left(i_{2}^{-1}(x)\right) & \text { for } x \in i_{2}\left(X_{2}\right) .\end{cases}
$$

Then $\tilde{g}$ is a pseudo-metric on the pseudo-bundle $\pi_{1} \cup_{(\tilde{f}, f)} \pi_{2}: V_{1} \cup_{\tilde{f}} V_{2} \rightarrow X_{1} \cup_{f} X_{2}$.

\footnotetext{
${ }^{5}$ Admittedly, I would not know what to do with a more general case.

${ }^{6}$ Recall that a map $h: X \rightarrow Y$ between two diffeological spaces is called an induction if it is injective, and the subset diffeology on $h(X) \subseteq Y$ coincides with the pushforward of the diffeology of $X$ by the map $h$.
} 


\section{Diffeological vector pseudo-bundles of Clifford algebras}

In this section we consider a finite-dimensional diffeological vector pseudo-bundle $\pi: V \rightarrow X$ carrying a pseudo-metric $g$. As we have shown above, this cannot be an arbitrary pseudo-bundle, since there are some that do not admit any pseudo-metric. Furthermore, as things stand now, it makes sense for us to limit this discussion to those pseudo-bundles that are obtained by gluing together some diffeologically trivial pseudo-bundles, usually ones of differing dimensions and along maps that may or may not be diffeomorphisms.

\subsection{Defining the pseudo-bundle of Clifford algebras $C \ell(V, g)$}

Let $\pi: V \rightarrow X$ be a finite-dimensional diffeological pseudo-bundle endowed with a pseudo-metric $g$ : $X \rightarrow V^{*} \otimes V^{*}$. Then there is a rather evident construction of the corresponding pseudo-bundle of Clifford algebras. We go over it as briefly as possible, since all the operations involved (direct sum, tensor product, and taking quotients), and their relevant properties 7 have already been described.

Definition 5.1. The pseudo-bundle of Clifford algebras $\pi^{C \ell}: C \ell(V, g) \rightarrow X$ is the space

$$
C \ell(V, g):=\bigcup_{x \in X} C \ell\left(\pi^{-1}(x), g(x)\right)
$$

equipped with the obvious projection $\pi^{C \ell}$ to $X$ and endowed with the finest diffeology such that the subset diffeology on each fibre $C \ell\left(\pi^{-1}(x), g(x)\right)$ coincides with the diffeology of the Clifford algebra of the diffeological vector space $\pi^{-1}(x)$ with the pseudo-metric $g(x)$.

With the definition stated in such terms, we should of course explain why such diffeology exists. The proof of this coincides with the explicit construction of the diffeology in question, which is as follows.

Consider first the pseudo-bundle of tensor algebras $\pi^{T(V)}: T(V) \rightarrow X$, where

$$
T(V):=\bigcup_{x \in X} T\left(\pi^{-1}(x)\right)
$$

with each $T\left(\pi^{-1}(x)\right):=\bigoplus_{r}\left(\pi^{-1}(x)\right)^{\otimes r}$ being the usual tensor algebra of the diffeological vector space $\pi^{-1}(x)$. The space $T(V)$ is endowed with the vector space direct sum diffeology relative to the tensor product diffeology on each factor. By the properties of these diffeologies, the subset diffeology on each fibre of $T(V)$ is that of the tensor algebra of the diffeological vector space $\pi^{-1}(x)$.

Next, for each fibre $\left(\pi^{T(V)}\right)^{-1}(x)=T\left(\pi^{-1}(x)\right)$ of $T(V)$ let $W_{x}$ be the kernel of the universal map $T\left(\pi^{-1}(x)\right) \rightarrow C \ell\left(\pi^{-1}(x), g(x)\right)$. Then, as is generally the case, $W=\bigcup_{x \in X} W_{x} \subset T(V)$, with the subset diffeology relative this inclusion, is a sub-bundle of $T(V)$.

Finally, consider the quotient pseudo-bundle $T(V) / W$. By definition of the quotient pseudo-bundle, it has $C \ell\left(\pi^{-1}(x), g(x)\right)$ as fibre at $x$, and so it coincides with $C \ell(V, g)$ as defined above. It then follows from general properties of quotient pseudo-bundles that, relative to the quotient diffeology, the subset diffeology on each fibre is the diffeology of the Clifford algebra of the corresponding fibre $\pi^{-1}(x)$ of $V$.

\subsection{The pseudo-bundle $C \ell\left(V_{1} \cup_{\tilde{f}} V_{2}, \tilde{g}\right)$}

We now consider the behavior of pseudo-bundles of Clifford algebras under the operation of diffeological gluing. We only treat the case of two factors (which of course may serve as an inductive step for a longer sequence of gluings, but we do not go into any detail about this).

\subsubsection{Existence and smoothness of a gluing map for $C \ell\left(V_{1}, g_{1}\right)$ and $C \ell\left(V_{2}, g_{2}\right)$}

Suppose that we are given a gluing $(\tilde{f}, f)$ between the pseudo-bundles $\pi_{1}: V_{1} \rightarrow X_{1}$ and $\pi_{2}: V_{2} \rightarrow X_{2}$ (endowed with a pseudo-metric each). The existence of a natural gluing map between the corresponding

\footnotetext{
${ }^{7}$ This mainly means the commutativity between considering the subset diffeology on each fibre and performing a given operation on the individual fibre or, respectively, the entire pseudo-bundle.
} 
pseudo-bundles of Clifford algebras is based, more than on anything else, on the universal properties of Clifford algebras, together with the commutativity properties considered above. More precisely, we have the following.

Lemma 5.2. Let $\pi_{1}: V_{1} \rightarrow X_{1}$ and $\pi_{2}: V_{2} \rightarrow X_{2}$ be finite-dimensional diffeological vector pseudobundles, let $(\tilde{f}, f)$ be a pair of smooth maps defining a gluing between them, let $Y \subseteq X_{1}$ be the domain of definition of $f$, and let $g_{1}$ and $g_{2}$ be compatible pseudo-metrics on $V_{1}$ and $V_{2}$ respectively. Then there exists a well-defined map

$$
\tilde{F}^{C \ell}:\left(\pi_{1}^{C \ell}\right)^{-1}(Y) \rightarrow\left(\pi_{2}^{C \ell}\right)^{-1}(f(Y)),
$$

that covers $f$ and whose restriction onto each fibre is an algebra homomorphism.

Proof. The construction of the map $\tilde{F}^{C \ell}$ is the obvious one; on each fibre $\left(\pi_{1}^{C \ell}\right)^{-1}(y)$ it is given by extending $\left.\tilde{f}\right|_{\pi_{1}^{-1}(y)}$ by linearity and tensor product multiplicativity. That this gives a well-defined map

$$
C \ell\left(\pi_{1}^{-1}(y), g_{1}(y)\right) \rightarrow C \ell\left(\pi_{2}^{-1}(f(y)), g_{2}(f(y))\right)
$$

follows from the compatibility of the pseudo-metrics with respect to $g_{1}$ and $g_{2}$.

Thus, in order to be able to claim the existence of a well-defined gluing between $C \ell\left(V_{1}, g_{1}\right)$ and $C \ell\left(V_{2}, g_{2}\right)$, we only need to establish the following statement.

Proposition 5.3. The map $\tilde{F}^{C l}$ is smooth.

Proof. Let $p: U \rightarrow\left(\pi_{1}^{C \ell}\right)^{-1}(Y)$ be a plot; by definition of the diffeology on any Clifford algebra, it locally lifts to a plot $\tilde{p}: U \rightarrow T\left(\pi_{1}^{-1}(Y)\right)$ of $T\left(\pi_{1}^{-1}(Y)\right)$. Observe next that this lift is compatible with the action of $\tilde{F}^{C \ell}$, in the following sense. If $\tilde{F}^{T V}$ is the lift of $\tilde{F}^{C \ell}$ to an algebra homomorphism

$$
\tilde{F}^{T V}: T\left(\pi_{1}^{-1}(Y)\right) \rightarrow T\left(\pi_{2}^{-1}(f(Y))\right),
$$

and

$$
\begin{gathered}
\Pi_{1}: T\left(\pi_{1}^{-1}(Y)\right) \rightarrow C \ell\left(\pi_{1}^{-1}(Y),\left.g_{1}\right|_{Y}\right), \\
\Pi_{2}: T\left(\pi_{2}^{-1}(f(Y))\right) \rightarrow C \ell\left(\pi_{2}^{-1}(f(Y)),\left.g_{2}\right|_{f(Y)}\right)
\end{gathered}
$$

are the corresponding universal maps, then we have

$$
\Pi_{2} \circ \tilde{F}^{T V} \circ \tilde{p}=\tilde{F}^{C l} \circ p .
$$

In particular (this is a consequence of the properties of the pushforward diffeologies), the map $\tilde{F}^{C l}$ is smooth if and only if $\tilde{F}^{T V}$ is smooth.

Let us then show that $\tilde{F}^{T V}$ is smooth, that is, that $\tilde{F}^{T V} \circ \tilde{p}$ is a plot of $T\left(\pi_{2}^{-1}(f(Y))\right.$ ) (where $\tilde{p}$ can now be any plot of $T\left(\pi_{1}^{-1}(Y)\right)$; we do not have to make a reference to it being a lift of a plot of the corresponding Clifford algebras' bundle). We can assume that the domain of definition of $\tilde{p}$ is small enough so that, one, its range is contained in $\bigoplus_{r=0}^{n}\left(\pi_{1}^{-1}(Y)\right)^{\otimes r}$ for some $n$, and, two, $\tilde{p}=\bigoplus_{r=0}^{n} \tilde{p}_{i}$, where $\tilde{p}_{i}: U \rightarrow \underbrace{\left(\pi_{1}^{-1}(Y)\right) \otimes \ldots \otimes\left(\pi_{1}^{-1}(Y)\right)}_{i}$ is a plot of a single summand (the tensor product of $i$ copies of $\left.\pi_{1}^{-1}(Y)\right)$.

Obviously, the composition $\tilde{F}^{T V} \circ \tilde{p}$ also splits as the analogous sum of the compositions $\bigoplus_{i} \tilde{F}^{T V} \circ \tilde{p}_{i}$, with range a single summand $\left(\pi_{2}^{-1}(f(Y))\right) \otimes \ldots \otimes\left(\pi_{2}^{-1}(f(Y))\right)$, so it suffices to show that each $\tilde{F}^{T V} \circ \tilde{p}_{i}$ is a plot of this tensor product. But by definition $\tilde{F}^{T V}$ preserves the tensor product, and each individual factor it coincides with (the appropriate restriction of) $\tilde{f}$. Thus, we conclude that each $\tilde{F}^{T V} \circ \tilde{p}_{i}$

$$
\tilde{F}^{T V} \circ \tilde{p}_{i}=(\underbrace{\tilde{f} \otimes \ldots \otimes \tilde{f}}_{i}) \circ \tilde{p}_{i} .
$$

Since the tensor product of smooth maps is smooth, this composition is indeed a plot of $\left(\pi_{2}^{-1}(f(Y))\right) \otimes$ $\ldots \otimes\left(\pi_{2}^{-1}(f(Y))\right)$. This allows us to conclude that $\tilde{F}^{T V}$ is smooth, and therefore so is $\tilde{F}^{C \ell}$. 
Essentially, what we need Proposition 5.3 for, is to be able to claim that there is a natural gluing of $\pi_{1}^{C \ell}: C \ell\left(V_{1}, g_{1}\right) \rightarrow X_{1}$ to $\pi_{2}^{C \ell}: C \ell\left(V_{2}, g_{2}\right) \rightarrow X_{2}$, which is along the maps $\tilde{F}^{C \ell}$ and $f$. Furthermore, still by compatibility between $g_{1}$ and $g_{2}$, one can consider the pseudo-bundle of Clifford algebras associated to $\left(V_{1} \cup_{\tilde{f}} V_{2}, \tilde{g}\right)$, that is, the pseudo-bundle

$$
\left(\pi_{1} \cup_{(\tilde{f}, f)} \pi_{2}\right)^{C \ell}: C \ell\left(V_{1} \cup_{\tilde{f}} V_{2}, \tilde{g}\right) \rightarrow X_{1} \cup_{f} X_{2} .
$$

We now make a comparison between these two pseudo-bundles, i.e., between

$$
\pi_{1}^{C \ell} \cup_{\left(\tilde{F}^{C \ell}, f\right)} \pi_{2}^{C \ell}: C \ell\left(V_{1}, g_{1}\right) \cup_{\tilde{F} C \ell} C \ell\left(V_{2}, g_{2}\right) \rightarrow X_{1} \cup_{f} X_{2}
$$

and

$$
\left(\pi_{1} \cup_{(\tilde{f}, f)} \pi_{2}\right)^{C \ell}: C \ell\left(V_{1} \cup_{\tilde{f}} V_{2}, \tilde{g}\right) \rightarrow X_{1} \cup_{f} X_{2},
$$

starting with the simpler case: when $\tilde{f}$ is a diffeomorphism.

\subsubsection{If $\tilde{f}$ is a diffeomorphism}

Expectedly, in this case the gluing of $C \ell\left(V_{1}, g_{1}\right)$ to $C \ell\left(V_{2}, g_{2}\right)$ along $\left(\tilde{F}^{C \ell}, f\right)$ yields a pseudo-bundle which is naturally diffeomorphic to the pseudo-bundle $C \ell\left(V_{1} \cup_{\tilde{f}} V_{2}, \tilde{g}\right)$. This is stated in the following theorem; notice that on the level of pseudo-metrics this is guaranteed by the fact that on each individual fibre, $\tilde{g}$ coincides with either $g_{1}$ or $g_{2}$, as appropriate.

Theorem 5.4. Let $\pi_{1}: V_{1} \rightarrow X_{1}$ and $\pi_{2}: V_{2} \rightarrow X_{2}$ be two finite-dimensional diffeological vector pseudo-bundles, let $(\tilde{f}, f)$ be a gluing between them such that $\tilde{f}$ is a diffeomorphism, and $g_{1}$ and $g_{2}$ be pseudo-metrics on $V_{1}$ and $V_{2}$ compatible with this gluing. Let $\pi_{i}^{C \ell}: C \ell\left(V_{i}, g_{i}\right) \rightarrow X_{i}$ for $i=1,2$ be the pseudo-bundle of Clifford algebras. Then the above-defined map

$$
\tilde{F}^{C \ell}:\left(\pi_{1}^{C \ell}\right)^{-1}(Y) \rightarrow\left(\pi_{2}^{C \ell}\right)^{-1}(f(Y))
$$

is such that the following two pseudo-bundles are diffeomorphic:

$$
\pi_{1}^{C \ell} \cup_{\left(\tilde{F}^{C \ell}, f\right)} \pi_{2}^{C \ell}: C \ell\left(V_{1}, g_{1}\right) \cup_{\tilde{F}^{C \ell}} C \ell\left(V_{2}, g_{2}\right) \rightarrow X_{1} \cup_{f} X_{2},
$$

and

$$
\left(\pi_{1} \cup_{(\tilde{f}, f)} \pi_{2}\right)^{C \ell}: C \ell\left(V_{1} \cup_{\tilde{f}} V_{2}, \tilde{g}\right) \rightarrow X_{1} \cup_{f} X_{2},
$$

where $\tilde{g}$ is the pseudo-metric on the pseudo-bundle

$$
\pi_{1} \cup_{(\tilde{f}, f)} \pi_{2}: V_{1} \cup_{\tilde{f}} V_{2} \rightarrow X_{1} \cup_{f} X_{2}
$$

induced by the pseudo-metrics $g_{1}$ and $g_{2}$.

We denote the diffeomorphism whose existence is claim in the theorem, by

$$
\Phi^{C \ell}: C \ell\left(V_{1}, g_{1}\right) \cup_{\tilde{F}}^{C \ell} C \ell\left(V_{2}, g_{2}\right) \rightarrow C \ell\left(V_{1} \cup_{\tilde{f}} V_{2}, \tilde{g}\right) .
$$

Proof. Since by construction $\tilde{F}^{C \ell}$ is an algebra homomorphism on each fibre, it is in particular linear; also by construction, it is a lift of $f$, so it does make sense to speak of gluing of the pseudo-bundles $\pi_{1}^{C \ell}$ : $C \ell\left(V_{1}, g_{1}\right) \rightarrow X_{1}$ and $\pi_{2}^{C \ell}: C \ell\left(V_{2}, g_{2}\right) \rightarrow X_{2}$ along $\tilde{F}^{C \ell}$ and $f$. We thus need to exhibit a diffeomorphism $\Phi^{C \ell}$ between $C \ell\left(V_{1}, g_{1}\right) \cup_{\tilde{F}^{C \ell}} C \ell\left(V_{2}, g_{2}\right)$ and $C \ell\left(V_{1} \cup_{\tilde{f}} V_{2}, \tilde{g}\right)$ covering the identity diffeomorphism on $X_{1} \cup_{f}$ $X_{2}$.

The existence of a diffeomorphism

$$
\Phi^{C \ell}: C \ell\left(V_{1}, g_{1}\right) \cup_{\tilde{F} C \ell} C \ell\left(V_{2}, g_{2}\right) \rightarrow C \ell\left(V_{1} \cup_{\tilde{f}} V_{2}, \tilde{g}\right)
$$

covering the identity map $X_{1} \cup_{f} X_{2} \rightarrow X_{1} \cup_{f} X_{2}$ can now be deduced from the construction of $\tilde{g}$, that of the map $\tilde{F}^{C \ell}$, as well as the commutativity between the gluing and the operations of the direct sum and 
tensor product (see Section 4.2.2). Specifically, applying the considerations already made to establish the smoothness of $\tilde{F}^{C l}$ and the just-mentioned commutativity, one immediately obtains a diffeomorphism

$$
\Phi^{T V}: T\left(V_{1}\right) \cup_{\tilde{F}^{T V}} T\left(V_{2}\right) \rightarrow T\left(V_{1} \cup_{\tilde{f}} V_{2}\right)
$$

that covers the identity map on $X_{1} \cup_{f} X_{2}$.

It then suffices to establish that the pushforward of $\Phi^{T V}$ by the natural projections

$$
\pi_{1}^{T V} \cup_{\left(\tilde{F}^{T V}, \tilde{F}^{C \ell}\right)} \pi_{2}^{T V}: T\left(V_{1}\right) \cup_{\tilde{F}^{T V}} T\left(V_{2}\right) \rightarrow C \ell\left(V_{1}, g_{1}\right) \cup_{\tilde{F}^{C \ell}} C \ell\left(V_{2}, g_{2}\right)
$$

and

$$
\pi^{T V}: T\left(V_{1} \cup_{\tilde{f}} V_{2}\right) \rightarrow C \ell\left(V_{1} \cup_{\tilde{f}} V_{2}, \tilde{g}\right)
$$

is well-defined and bijective; the diffeologies on the ranges being pushforward diffeologies — by these same projections -, the smoothness is then automatic. That it is well-defined follows from the fact that $\Phi^{T V}$ sends the kernel of $\pi_{1}^{T V} \cup_{\left(\tilde{F}^{T V}, \tilde{F}^{C \ell}\right)} \pi_{2}^{T V}$ to the kernel of $\pi^{T V}$. That it is bijective is the result of the fact that the map $\Phi^{T V}$ is so (and this is because $\tilde{f}$ is a diffeomorphism to start with), and we obtain the claim

Finally, observe that from this theorem, it is quite easy to extrapolate its extension to the case of more consecutive gluings, as long as they are all done along diffeomorphisms.

\subsubsection{Non-diffeomorphism $\tilde{f}$}

We now briefly consider what happens if the assumption of $\tilde{f}$ being a diffeomorphism is dropped. Of course, if $\tilde{f}$ is not a diffeomorphism then neither is $\tilde{F}^{C \ell}$. However, what we are really interested in is the relation between $C \ell\left(V_{1}, g_{1}\right) \cup_{\tilde{F} C \ell} C \ell\left(V_{2}, g_{2}\right)$ and $C \ell\left(V_{1} \cup_{\tilde{f}} V_{2}, \tilde{g}\right)$, where, as we are about to explain, the main point is the commutativity of the gluing operation with those of the direct sum and the tensor product of pseudo-bundles, rather than the specific properties of the gluing map.

The map $\Phi^{T V}$ As we have already observed, the properties of the map $\tilde{F}^{C l}$ are largely defined by those of its lift $\tilde{F}^{T V}: T\left(V_{1}\right) \rightarrow T\left(V_{2}\right)$, and the previous construction of the diffeomorphism

$$
\Phi^{C \ell}: C \ell\left(V_{1}, g_{1}\right) \cup_{\tilde{F}^{C \ell}} C \ell\left(V_{2}, g_{2}\right) \rightarrow C \ell\left(V_{1} \cup_{\tilde{f}} V_{2}, \tilde{g}\right)
$$

was by pushing forward the then-diffeomorphism $\Phi^{T V}: T\left(V_{1}\right) \cup_{\tilde{F}^{T V}} T\left(V_{2}\right) \rightarrow T\left(V_{1} \cup_{\tilde{f}} V_{2}\right)$.

Lemma 5.5. The map $\Phi^{T V}: T\left(V_{1}\right) \cup_{\tilde{F}^{T V}} T\left(V_{2}\right) \rightarrow T\left(V_{1} \cup_{\tilde{f}} V_{2}\right)$ is a diffeomorphism.

The difference of this statement with respect to what was claimed, regarding the map $\Phi^{T V}$, in the proof of Theorem 5.4 is the absence of any assumption on $\tilde{f}$ (when it was a diffeomorphism, we essentially took this statement for granted).

Proof. The claim follows from the commutativity between gluing, and the operations of direct sum and tensor product. In particular, let us show that $\Phi^{T V}$ is bijective. In the formulas below, we us the notation $V_{x}$ for the fibre at $x$ of any pseudo-bundle $V$.

Consider the $r$-th summand in the definition of, respectively,

$$
\begin{aligned}
& T\left(V_{1}\right)=\bigoplus_{k} V_{1}^{\otimes k}=\bigcup_{x_{1} \in X_{1}} \bigoplus_{k}\left(V_{1}^{\otimes k}\right)_{x_{1}}=\bigcup_{x_{1} \in X_{1}} \bigoplus_{k}\left(\left(V_{1}\right)_{x_{1}}\right)^{\otimes k}, \\
& T\left(V_{2}\right)=\bigoplus_{k} V_{2}^{\otimes k}=\bigcup_{x_{2} \in X_{2}} \bigoplus_{k}\left(V_{2}^{\otimes k}\right)_{x_{2}}=\bigcup_{x_{2} \in X_{2}} \bigoplus_{k}\left(\left(V_{2}\right)_{x_{2}}\right)^{\otimes k},
\end{aligned}
$$

and

$$
T\left(V_{1} \cup_{\tilde{f}} V_{2}\right)=\bigoplus_{k}\left(V_{1} \cup_{\tilde{f}} V_{2}\right)^{\otimes k}=\bigcup_{x \in X_{1} \cup_{f} X_{2}} \bigoplus_{k}\left(\left(V_{1} \cup_{\tilde{f}} V_{2}\right)^{\otimes k}\right)_{x}=\bigcup_{x \in X_{1} \cup_{f} X_{2}} \bigoplus_{k}\left(\left(V_{1} \cup_{\tilde{f}} V_{2}\right)_{x}\right)^{\otimes k} .
$$


Observe first of all that, by its definition, the map $\tilde{F}^{T V}$ (in addition to being fibre-to-fibre) sends the $r$ th summand of the first direct sum to the $r$ th summand of the second direct sum,

$$
\tilde{F}^{T V}\left(\left(\left(V_{1}\right)_{y}\right)^{\otimes r}\right) \subseteq\left(\left(V_{2}\right)_{f(y)}\right)^{\otimes r} \text { for all } y \in Y .
$$

Furthermore, within each tensor product it acts as the tensor product of $r$ times $\tilde{f}$ with itself. The fibre over $i_{2}(f(y))$ of $T\left(V_{1}\right) \cup_{\tilde{F}^{T V}} T\left(V_{2}\right)$ is therefore

$$
\left(\left(\left(V_{1}\right)_{y}\right)^{\otimes r}\right) \underbrace{\tilde{f} \otimes \ldots \otimes \tilde{f}}_{r}\left(\left(\left(V_{2}\right)_{f(y)}\right)^{\otimes r}\right) .
$$

Since gluing commutes with tensor product, it is quite easy to see that this is the same as the corresponding fibre of $T\left(V_{1} \cup_{\tilde{f}} V_{2}\right)$.

Finally, the fact that $\Phi^{T V}$ is smooth follows from its construction, and the definitions of the diffeologies involved. Indeed, by definition of a vector space's direct sum diffeology, $\Phi^{T V}$ is smooth if and only if its restriction on the corresponding direct sum factors (i.e., on all individual tensor products and, for the domain, their gluings) is smooth. That such restriction is smooth for each $k$ is the essence of the commutativity between the gluing and the tensor product stated in Section 4.2.2.

The map $\Phi^{C l} \quad$ Having established that $\Phi^{T V}$ being a diffeomorphism does not depend on $\tilde{f}$ being, or not being, one, we now draw the analogous conclusion for the pseudo-bundles of Clifford algebras. This can now also be done in the manner completely analogous to the proof of Theorem 5.4.

In the theorem below we assume that $\pi_{1}: V_{1} \rightarrow X_{1}, \pi_{2}: V_{2} \rightarrow X_{2}, g_{1}$, and $g_{2}$ are as in Theorem 5.4, but the gluing pair $(\tilde{f}, f)$ is arbitrary (assuming that it is suitable for gluing, of course). Also notice that the fact of the existence of pseudo-metrics compatible with it does impose significant restrictions on $\tilde{f}$ (which, on the other hand, may still have a non-trivial kernel, see [10]).

Theorem 5.6. There is a diffeomorphism $\Phi^{C \ell}: C \ell\left(V_{1}, g_{1}\right) \cup_{\tilde{F}}^{C \ell} C \ell\left(V_{2}, g_{2}\right) \rightarrow C \ell\left(V_{1} \cup_{\tilde{f}} V_{2}, \tilde{g}\right)$.

Proof. Obviously, $\Phi^{C l}$ is obtained, as before, by pushing forward $\Phi^{T V}$ via the natural projections. It is also smooth for all the same reasons as in the previous case (these reasons are, it is obtained via a pushforward of a smooth map, and the diffeologies on its domain and its range are pushforward diffeologies as well).

That $\Phi^{C l}$ is bijective, follows from examining the structure of individual fibres in the two spaces. The main argument is that over a point in $i_{2}(f(y))$ for $y \in Y$, the fibre in the domain of $\Phi^{C l}$ is naturally diffeomorphic to $C \ell\left(\pi_{2}^{-1}(f(y)), g_{2}\right)$, which follows from the definition of gluing, whereas, since the fibre over $i_{2}(f(y))$ in $V_{1} \cup_{\tilde{f}} V_{2}$ is $j_{2}\left(\pi_{2}^{-1}(f(y))\right)$, the corresponding fibre in the range of $\Phi^{C l}$ is equal to $C \ell\left(\pi_{2}^{-1}(f(y)),\left.\tilde{g}\right|_{\pi_{2}^{-1}(f(y))}\right)=C \ell\left(\pi_{2}^{-1}(f(y)), g_{2}\right)$ (this is by definition of $\left.\tilde{g}\right)$. It remains to notice that the action of $\Phi^{T V}$ and then that of $\Phi^{C \ell}$ corresponds each to the identity under these diffeomorphisms.

\section{The pseudo-bundles of Clifford modules}

In this concluding section we consider the behavior of the pseudo-bundles of Clifford modules under the operation of gluing. Let $X_{1}$ and $X_{2}$ be two diffeological spaces, and let $f: X_{1} \supset Y \rightarrow X_{2}$ be a smooth map. Consider two finite-dimensional diffeological vector pseudo-bundles over them, $\pi_{1}: V_{1} \rightarrow X_{1}$ and $\pi_{2}: V_{2} \rightarrow X_{2}$, and a smooth fibrewise-linear lift $\tilde{f}: \pi_{1}^{-1}(Y) \rightarrow \pi_{2}^{-1}(f(Y))$ of $f$. Suppose that each of these pseudo-bundles carries a pseudo-metric, $g_{1}$ and $g_{2}$ respectively, and suppose that these pseudo-metrics are compatible for the gluing defined by $\tilde{f}$ and $f$; consider the corresponding pseudo-bundles of Clifford algebras,

$$
\pi_{1}^{C \ell}: C \ell\left(V_{1}, g_{1}\right) \rightarrow X_{1} \quad \text { and } \quad \pi_{2}^{C \ell}: C \ell\left(V_{2}, g_{2}\right) \rightarrow X_{2},
$$

and the pseudo-bundle obtained by gluing

$$
C \ell\left(V_{1}, g_{1}\right) \cup_{\tilde{F} C \ell} C \ell\left(V_{2}, g_{2}\right)=C \ell\left(V_{1} \cup_{\tilde{f}} V_{2}, \tilde{g}\right) \rightarrow X_{1} \cup_{f} X_{2} .
$$


Suppose furthermore that we are given two pseudo-bundles of Clifford modules,

$$
\chi_{1}: E_{1} \rightarrow X_{1} \text { and } \chi_{2}: E_{2} \rightarrow X_{2},
$$

that is, for $i=1,2$ there is a smooth pseudo-bundle map

$$
c_{i}: C \ell\left(V_{i}, g_{i}\right) \rightarrow \mathcal{L}\left(E_{i}, E_{i}\right)
$$

that covers the identity on the bases. Suppose finally that there is a smooth fibrewise linear map

$$
\tilde{f}^{\prime}: \chi_{1}^{-1}(Y) \rightarrow \chi_{2}^{-1}(f(Y))
$$

that covers $f$. We wish to specify under which conditions $E_{1} \cup_{\tilde{f}} E_{2}$ is a Clifford module over $C \ell\left(V_{1} \cup_{\tilde{f}} V_{2}, \tilde{g}\right)$, via an action induced by $c_{1}$ and $c_{2}$.

Notation Below we will deal with more than one pair of gluings of pseudo-bundles at a time; to be able to distinguish between them, we now introduce a somewhat more complicated version of the notation for the inductions $j_{1}$ and $j_{2}$. Specifically, we denote

$$
j_{1}^{C \ell}: C \ell\left(V_{1}, g_{1}\right) \backslash\left(\pi_{1}^{C \ell}\right)^{-1}(Y) \hookrightarrow C \ell\left(V_{1}, g_{1}\right) \cup_{\tilde{F}^{C \ell}} C \ell\left(V_{2}, g_{2}\right)
$$

and

$$
j_{2}^{C \ell}: C \ell\left(V_{2}, g_{2}\right) \hookrightarrow C \ell\left(V_{1}, g_{1}\right) \cup_{\tilde{F} C \ell} C \ell\left(V_{2}, g_{2}\right)
$$

the two standard inductions for the pseudo-bundles $C \ell\left(V_{1}, g_{1}\right) \cup_{\tilde{F} C \ell} C \ell\left(V_{2}, g_{2}\right)$. In contrast, we denote by

$$
j_{1}^{E_{1}}: E_{1} \backslash \chi_{1}^{-1}(Y) \rightarrow E_{1} \cup_{\tilde{f}^{\prime}} E_{2}
$$

and

$$
j_{2}^{E_{2}}: E_{2} \hookrightarrow E_{1} \cup_{\tilde{f}^{\prime}} E_{2}
$$

the two standard inductions for the pseudo-bundle $E_{1} \cup_{\tilde{f}} E_{2}$.

Compatibility of $c_{1}$ and $c_{2}$ Let $y \in Y$, and let $v \in\left(\pi_{1}^{C \ell}\right)^{-1}(y)$. Consider the element

$$
\Phi^{C \ell}\left(j_{2}^{C \ell}\left(\tilde{F}^{C \ell}(v)\right)\right) \in\left(\left(\pi_{1} \cup_{(\tilde{f}, f)} \pi_{2}\right)^{C \ell}\right)^{-1}\left(i_{2}(f(y))\right) \subset C \ell\left(V_{1} \cup_{\tilde{f}} V_{2}, \tilde{g}\right) ;
$$

the two relevant 8 actions are

$$
c_{1}(v):\left(E_{1}\right)_{y} \rightarrow\left(E_{1}\right)_{y}, \text { and } c_{2}\left(\tilde{F}^{C \ell}(v)\right):\left(E_{2}\right)_{f(y)} \rightarrow\left(E_{2}\right)_{f(y)} .
$$

We shall now compare the following two:

$$
\tilde{f}^{\prime} \circ\left(c_{1}(v)\right), \text { and }\left(c_{2}\left(\tilde{F}^{C \ell}(v)\right)\right) \circ \tilde{f}^{\prime} .
$$

Our aim is to obtain a well-defined action on $E_{1} \cup_{\tilde{f}^{\prime}} E_{2}$, and a potential issue presents itself precisely for elements of form $\Phi^{C \ell}\left(j_{2}^{C \ell}\left(\tilde{F}^{C \ell}(v)\right)\right)$. Indeed, this element acts on the fibre $\left(E_{1} \cup_{\tilde{f}^{\prime}} E_{2}\right)_{i_{2}(f(y))}$. Its action therefore can potentially be described by

$$
\Phi^{C \ell}\left(j_{2}^{C \ell}\left(\tilde{F}^{C \ell}(v)\right)\right)\left(\tilde{f}^{\prime}\left(e_{1}\right)\right)=\tilde{f}^{\prime}\left(c_{1}(v)\left(e_{1}\right)\right) \text { for any } e_{1} \in\left(E_{1}\right)_{y} .
$$

On the other hand, for a generic element $e_{2} \in\left(E_{2}\right)_{f(y)}$ (generic meaning that it does not have to belong to the image of $\tilde{f}^{\prime}$ ) we might have

$$
\Phi^{C \ell}\left(j_{2}^{C \ell}\left(\tilde{F}^{C \ell}(v)\right)\right)\left(e_{2}\right)=c_{2}\left(\tilde{F}^{C \ell}(v)\right)\left(e_{2}\right) .
$$

In order to obtain a smooth induced action, we wish to ensure that these expressions are compatible with each other. We thus obtain the following notion.

Definition 6.1. The actions $c_{1}$ and $c_{2}$ are compatible if for all $y \in Y$, for all $v \in\left(\pi_{1}^{C \ell}\right)^{-1}(y)$, and for all $e_{1} \in \chi_{1}^{-1}(y)=\left(E_{1}\right)_{y}$ we have

$$
\tilde{f}^{\prime}\left(c_{1}(v)\left(e_{1}\right)\right)=c_{2}\left(\tilde{F}^{C \ell}(v)\right)\left(\tilde{f}^{\prime}\left(e_{1}\right)\right)
$$

\footnotetext{
${ }^{8}$ In the sense of the two points in the base that come into play here.
} 
Compatibility of actions as an instance of compatibility of maps Let us now see whether the notion of two compatible actions is an instance of the usual $(f, g)$-compatibility for smooth maps between diffeological spaces (see [9], Section 4.2). Each $c_{i}$ can be seen as a pseudo-bundle map $c_{i}: C \ell\left(V_{i}, g_{i}\right) \rightarrow$ $\mathcal{L}\left(E_{i}, E_{i}\right)$; on the relevant subset of $C \ell\left(V_{1}, g_{1}\right)$ (this subset is $\left.C \ell\left(\pi_{1}^{-1}(Y),\left.g_{1}\right|_{Y}\right)\right)$ we have the map $\tilde{F}^{C \ell}$. Let us consider the possibility of there being a lift $\tilde{F}^{\text {mod }}$ of $\tilde{F}^{C \ell}$ for the corresponding subsets of $\mathcal{L}\left(E_{1}, E_{1}\right)$ and $\mathcal{L}\left(E_{2}, E_{2}\right)$.

This prospective lift, first of all, should act between the restricted pseudo-bundles, namely, between $\bigcup_{y \in Y} L^{\infty}\left(\left(E_{1}\right)_{y},\left(E_{1}\right)_{y}\right)$ and $\bigcup_{y^{\prime} \in f(Y)} L^{\infty}\left(\left(E_{2}\right)_{y^{\prime}},\left(E_{2}\right)_{y^{\prime}}\right)$. It should be noticed at this point that the possibility to define the desired lift from the actions $c_{1}$ and $c_{2}$ is not guaranteed, since the image of $c_{1}$ may not include whole fibres over $Y$, meaning, more precisely, that for a given $y \in Y$ the image of the restriction of $c_{1}$ to $C \ell\left(\left(V_{1}\right)_{y}, g_{1}(y)\right)$ is a subset (and a subspace, of course) of $L^{\infty}\left(\left(E_{1}\right)_{y},\left(E_{1}\right)_{y}\right)$ (the fibre at $y$ of $\left.\mathcal{L}\left(E_{1}, E_{1}\right)\right)$, but it may be a proper subspace. Now, what we want of

$$
\tilde{F}^{\text {mod }}: \mathcal{L}\left(E_{1}, E_{1}\right) \supset\left(\pi_{1}^{\mathcal{L}}\right)^{-1}(Y) \rightarrow\left(\pi_{2}^{\mathcal{L}}\right)^{-1}(f(Y)) \subset \mathcal{L}\left(E_{2}, E_{2}\right)
$$

is that for every $y \in Y$ and for every $v \in\left(\pi_{1}^{C \ell}\right)^{-1}(y)$ there be the equality

$$
\tilde{F}^{m o d}\left(c_{1}(v)\right)=c_{2}\left(\tilde{F}^{C \ell}(v)\right)
$$

and if $\left.c_{1}\right|_{C \ell\left(\left(V_{1}\right)_{y}, g_{1}(y)\right)}$ is not surjective, this equality is not sufficient to determine $\tilde{F}^{\text {mod }}$.

The induced action The above considerations however do not prevent us from defining an induced action, meaning a homomorphism

$$
c: C \ell\left(V_{1} \cup_{\tilde{f}} V_{2}, \tilde{g}\right) \rightarrow \mathcal{L}\left(E_{1} \cup_{\tilde{f}^{\prime}} E_{2}, E_{1} \cup_{\tilde{f}^{\prime}} E_{2}\right) .
$$

Recalling the above identification by the diffeomorphism $\left(\Phi^{C \ell}\right)^{-1}$

$$
C \ell\left(V_{1} \cup_{\tilde{f}} V_{2}, \tilde{g}\right) \cong C \ell\left(V_{1}, g_{1}\right) \cup_{\tilde{F}} C \ell\left(V_{2}, g_{2}\right),
$$

the action $c$ can be described as follows:

$$
c(v)(e)= \begin{cases}j_{1}^{E_{1}}\left(c_{1}\left(\left(j_{1}^{C \ell}\right)^{-1}(v)\right)\left(\left(j_{1}^{E_{1}}\right)^{-1}(e)\right)\right) & \text { if } v \in \operatorname{Im}\left(j_{1}^{C \ell}\right) \Rightarrow e \in \operatorname{Im}\left(j_{1}^{E_{1}}\right), \\ j_{2}^{E_{2}}\left(c_{2}\left(\left(j_{2}^{C \ell}\right)^{-1}(v)\right)\left(\left(j_{2}^{E_{2}}\right)^{-1}(e)\right)\right) & \text { if } v \in \operatorname{Im}\left(j_{2}^{C \ell}\right) \Rightarrow e \in \operatorname{Im}\left(j_{2}^{E_{2}}\right) .\end{cases}
$$

Since the images of the inductions $j_{1}^{C \ell}, j_{2}^{C \ell}$ are disjoint and cover $C \ell\left(V_{1}, g_{1}\right) \cup_{\tilde{F}} C \ell\left(V_{2}, g_{2}\right)$, and those of $j_{1}^{E_{1}}, j_{2}^{E_{2}}$ cover $E_{1} \cup_{\tilde{f}}, E_{2}$ (and are disjoint as well), this action is well-defined. Furthermore, each $c(v)$ is an endomorphism of the corresponding fibre, because both $c_{1}\left(\left(j_{1}^{C \ell}\right)^{-1}(v)\right)$ and $c_{2}\left(\left(j_{2}^{C \ell}\right)^{-1}(v)\right.$ ) (whichever is relevant) are so.

By construction, $c$ is an action of $C \ell\left(V_{1}, g_{1}\right) \cup_{\tilde{F}} C \ell\left(V_{2}, g_{2}\right)$; to obtain that of the diffeomorphic $C \ell\left(V_{1} \cup_{\tilde{f}}\right.$ $\left.V_{2}, \tilde{g}\right)$, it suffices to pre-compose $c$ with the diffeomorphism $\left(\Phi^{C \ell}\right)^{-1}$. In other words, it suffices to substitute $v$ in the above formula with $\left(\Phi^{C \ell}\right)^{-1}\left(v^{\prime}\right)$ for any $v^{\prime} \in C \ell\left(V_{1} \cup_{\tilde{f}} V_{2}, \tilde{g}\right)$.

Theorem 6.2. The action $c$ is smooth as a map

$$
C \ell\left(V_{1}, g_{1}\right) \cup_{\tilde{F}} C \ell\left(V_{2}, g_{2}\right) \rightarrow \mathcal{L}\left(E_{1} \cup_{\tilde{f}^{\prime}} E_{2}, E_{1} \cup_{\tilde{f}^{\prime}} E_{2}\right) .
$$

Proof. Let $p: U \rightarrow C \ell\left(V_{1}, g_{1}\right) \cup_{\tilde{F}} C \ell\left(V_{2}, g_{2}\right)$ be a plot; we need to show that $U \ni u \mapsto c(p(u))$ is a plot of $\mathcal{L}\left(E_{1} \cup_{\tilde{f}^{\prime}} E_{2}, E_{1} \cup_{\tilde{f}^{\prime}} E_{2}\right)$. Recall that the latter means that for any plot $q: U^{\prime} \rightarrow E_{1} \cup_{\tilde{f}^{\prime}} E_{2}$ the evaluation map $\left(u, u^{\prime}\right) \mapsto c(p(u))\left(q\left(u^{\prime}\right)\right) \in E_{1} \cup_{\tilde{f}^{\prime}} E_{2}$ is smooth, for the subset diffeology of the subset of $U \times U^{\prime}$ on which it is defined.

Take an arbitrary $q$ and recall that locally it lifts to either a plot $q_{1}$ of $E_{1}$ or a plot $q_{2}$ of $E_{2}$. In the first case $q$ has form

$$
q\left(u^{\prime}\right)= \begin{cases}j_{1}^{E_{1}}\left(q_{1}\left(u^{\prime}\right)\right) & \text { if } q_{1}\left(u^{\prime}\right) \in \chi_{1}^{-1}\left(X_{1} \backslash Y\right), \\ j_{2}^{E_{2}}\left(\tilde{f}^{\prime}\left(q_{1}\left(u^{\prime}\right)\right)\right) & \text { if } q_{1}\left(u^{\prime}\right) \in \chi_{1}^{-1}(Y)\end{cases}
$$


In the second case $q$ has form $q=j_{2}^{E_{2}} \circ q_{2}$. For each of these, we need to show that $c(p(u))\left(q\left(u^{\prime}\right)\right)$ is again a plot of the same (respective, since $c(\cdot)$ acts fibrewise) form.

Likewise, $p$ is a plot of $C \ell\left(V_{1}, g_{1}\right) \cup_{\tilde{F}} C \ell\left(V_{2}, g_{2}\right)$; therefore it also lifts to either a plot $p_{1}$ of $C \ell\left(V_{1}, g_{1}\right)$ or a plot $p_{2}$ of $C \ell\left(V_{2}, g_{2}\right)$. In the former case $p$ has form

$$
p(u)= \begin{cases}j_{1}^{C \ell}\left(p_{1}(u)\right) & \text { if } p_{1}(u) \notin\left(\pi_{1}^{C \ell}\right)^{-1}(Y) \\ j_{2}^{C \ell}\left(\tilde{F}^{C \ell}\left(p_{1}(u)\right)\right) & \text { if } p_{1}(u) \in\left(\pi_{1}^{C \ell}\right)^{-1}(Y)\end{cases}
$$

In the latter case we have $p=j_{2}^{C \ell} \circ p_{2}$. Notice furthermore that, if we assume the domain of the evaluation map to be small enough, then either $p$ lifts to $p_{1}$ and $q$ lifts to $q_{1}$ (but not to $q_{2}$ ), or $p$ lifts to $p_{2}$ and $q$ lifts to $q_{2} 9$ Thus, there are no more than two cases that determine the value of the evaluation map.

Let us consider the first case, i.e., where $p$ and $q$ lift to $p_{1}$ and $q_{1}$ respectively. Putting together the definition of the action $c$ (given prior to the statement of the proposition), and the descriptions of $p$ and $q$ for this case, we obtain:

$$
c(p(u))\left(q\left(u^{\prime}\right)\right)= \begin{cases}j_{1}^{E_{1}}\left(c_{1}\left(p_{1}(u)\right)\left(q_{1}\left(u^{\prime}\right)\right)\right) & \text { if } p_{1}(u) \notin\left(\pi_{1}^{C \ell}\right)^{-1}(Y), \\ j_{2}^{E_{2}}\left(c_{2}\left(\tilde{F}^{C \ell}\left(p_{1}(u)\right)\right)\left(\tilde{f}^{\prime}\left(q_{1}\left(u^{\prime}\right)\right)\right)\right) & \text { if } p_{1}(u) \in\left(\pi_{1}^{C \ell}\right)^{-1}(Y) .\end{cases}
$$

Recall that by the compatibility of the actions $c_{1}$ and $c_{2}$ we have

$$
j_{2}^{E_{2}}\left(c_{2}\left(\tilde{F}^{C \ell}\left(p_{1}(u)\right)\right)\left(\tilde{f}^{\prime}\left(q_{1}\left(u^{\prime}\right)\right)\right)\right)=j_{2}^{E_{2}}\left(\tilde{f}^{\prime}\left(c_{1}\left(p_{1}(u)\right)\left(q_{1}\left(u^{\prime}\right)\right)\right)\right) .
$$

Thus, it remains to observe that this is precisely one of the possible forms for a plot of $E_{1} \cup_{\tilde{f}^{\prime}} E_{2}$ (which is what we needed to obtain).

Let us now consider the second case, that of $p=j_{2}^{C \ell} \circ p_{2}$. Then it follows immediately from our definition of $c$ that

$$
c(p(u))\left(q\left(u^{\prime}\right)\right)=j_{2}^{E_{2}}\left(c_{2}\left(p_{2}(u)\right)\left(q_{2}\left(u^{\prime}\right)\right)\right),
$$

and the desired conclusion follows from the smoothness of $c_{2}$ and the fact that $j_{2}^{C l}$ is an induction. Since all the cases have thereby been considered, the statement is proven.

\section{$7 \quad$ An example}

We now briefly illustrate the constructions of the previous two sections, by considering the gluing presentation of a certain pseudo-bundle $\pi: V \rightarrow X$ underlying the internal tangent bundle $T^{d v s}(\hat{X})$ (see [1]) of the space $\hat{X}=\mathbb{R} \vee \mathbb{R}$. When the latter space is viewed as the union of the coordinate axes in $\mathbb{R}^{2}$ and endowed with the subset diffeology coming from the standard diffeology on $\mathbb{R}^{2}$, the resulting internal tangent bundle has the same fibrewise structure, but the diffeology on both the base space is stronger than that of our $X$, and the diffeology on the total space is stronger than the one on $V$. This follows, in particular, from an example in [16]).

\subsection{The pseudo-bundle $\pi: V \rightarrow X$}

We first describe our pseudo-bundle $\pi: V \rightarrow X$, immediately in terms of a gluing construction of it, and endow it with a pseudo-metric (corresponding to this gluing). We also specify how it is related to $T^{d v s}(\hat{X})$.

\subsubsection{The gluing presentation of $\pi: V \rightarrow X$}

The pseudo-bundle $\pi: V \rightarrow X$ is obtained by gluing together three other pseudo-bundles. The first of these is the pseudo-bundle $\pi_{0}: V_{0} \rightarrow X_{0}$, where $V_{0}$ is the standard $\mathbb{R}^{2}$ and $X_{0}=\left\{x_{0}\right\}$ is a one-point set. The second and the third pseudo-bundles are $\pi_{1}: V_{1} \rightarrow X_{1}$ and $\pi_{2}: V_{2} \rightarrow X_{2}$, where both $V_{1}$ and $V_{2}$ are the standard $\mathbb{R}^{2}$, and both $X_{1}$ and $X_{2}$ are the standard $\mathbb{R}$; for convenience, we write $\pi_{1}$ as the projection onto the first coordinate, $\pi_{1}(x, y)=x$, and $\pi_{2}$ as the projection onto the second coordinate, $\pi_{2}(x, y)=y$.

\footnotetext{
${ }^{9}$ This is the only place where we use the fact that $c$ acts between total spaces of pseudo-bundles.
} 
We then define the following two gluings, the first one of $V_{1}$ to $V_{0}$ and the second one of $V_{2}$ to $V_{0}$. These gluings are along the maps $\left(\tilde{f}_{1}, f_{1}\right)$ and $\left(\tilde{f}_{2}, f_{2}\right)$, where the maps $f_{1}:\{0\} \rightarrow\left\{x_{0}\right\}$ and $f_{2}:\{0\} \rightarrow\left\{x_{0}\right\}$ are the obvious ones, while $\tilde{f}_{1}$ and $\tilde{f}_{2}$ are the standard embeddings of the lines $\{(0, y)\}$ and $\{(x, 0)\}$ into $\mathbb{R}^{2}$, that is, $\tilde{f}_{1}: V_{1} \ni(0, y) \mapsto(0, y) \in V_{0}$ and $\tilde{f}_{2}: V_{2} \ni(x, 0) \mapsto(x, 0) \in V_{0}$. The pseudo-bundle $\pi: V \rightarrow X$ is obtained by first gluing $V_{1}$ to $V_{0}$ along $\left(\tilde{f}_{1}, f_{1}\right)$ and by then gluing $V_{2}$ to $V_{1} \cup_{\tilde{f}_{1}} V_{0}$ along $\left(j_{2}^{V_{0}} \circ \tilde{f}_{2}, i_{2} \circ f_{2}\right)$.

The resulting pseudo-bundle can also be described as follows:

$$
\left\{\begin{array}{l}
V=\{(x, y, z, w) \mid x y=0, x \neq 0 \Rightarrow w=0, y \neq 0 \Rightarrow z=0\} \subset \mathbb{R}^{4}, \\
X=\{(x, y, 0,0) \mid x y=0\} \subset \mathbb{R}^{4}, \\
\pi(x, y, w, z)=(x, y, 0,0) .
\end{array}\right.
$$

This presentation in accordance with the analogous presentations of $\pi_{0}: V_{0} \rightarrow X_{0}, \pi_{1}: V_{1} \rightarrow X_{1}$, and $\pi_{2}: V_{2} \rightarrow X_{2}$, which are as follows:

$$
\left\{\begin{array}{lll}
V_{0}=\{(0,0, z, w)\}, & X_{0}=\{(0,0,0,0)\}, & \pi_{0}=\left.\pi\right|_{V_{0}}, \\
V_{1}=\{(x, 0, z, 0)\}, & X_{1}=\{(x, 0,0,0)\}, & \pi_{1}=\left.\pi\right|_{V_{1}}, \\
V_{2}=\{(0, y, 0, w)\}, & X_{2}=\{(0, y, 0,0)\}, & \pi_{2}=\left.\pi\right|_{V_{2}} .
\end{array}\right.
$$

With respect to these presentations, the gluing maps assume the following form:

$$
\begin{cases}f_{1}(0,0,0,0)=(0,0,0,0) & f_{2}(0,0,0,0)=(0,0,0,0) \\ \tilde{f}_{1}(0,0, z, 0)=(0,0, z, 0) & \tilde{f}_{2}(0,0,0, w)=(0,0,0, w)\end{cases}
$$

\subsubsection{A corresponding pseudo-metric $\tilde{g}$}

To give a common description of the four pseudo-metrics that we will need, any one of those has the following shape. It is defined, first of all, on an appropriate subset of the set $\{(x, y, 0,0)\}$; to each such point it assigns a symmetric bilinear form on the set $\{(x, y, z, w)\}$ with $x, y$ fixed and $z, w$. Thus, in resemblance to the standard case, we will define our pseudo-metrics by quadratic forms in $d z$ and $d w$, with the following meaning:

$$
\left\{\begin{array}{l}
g^{\prime}(x, y, 0,0)=h_{11}(x, y) d z^{2}+h_{12}(x, y) d z d w+h_{22}(x, y) d w^{2} \\
g^{\prime}(x, y, 0,0)\left(\left(x, y, z_{1}, w_{1}\right),\left(x, y, z_{2} w_{2}\right)\right)=h_{11}(x, y) z_{1} z_{2}+h_{12}\left(z_{1} w_{2}+z_{2} w_{1}\right)+h_{22} w_{1} w_{2}
\end{array}\right.
$$

where $h_{11}, h_{12}, h_{22}: \mathbb{R}^{2} \rightarrow \mathbb{R}$ are some ordinary smooth functions, on which additional conditions are imposed, according to the specific case we are treating.

The pseudo-metrics $g_{0}, g_{1}$, and $g_{2}$ Using the notation just introduced, we define the following pseudo-metrics on $V_{1}, V_{2}$, and $V_{0}$, denoted respectively by $g_{0}, g_{1}$, and $g_{2}$ :

$$
\left\{\begin{array}{l}
g_{0}(0,0,0,0)=d z^{2}+d w^{2} \\
g_{1}(x, 0,0,0)=\left(x^{2}+1\right) d z^{2} \\
g_{2}(0, y, 0,0)=\left(y^{2}+1\right) d w^{2}
\end{array}\right.
$$

In particular, the compatibility of $g_{1}$ with $g_{0}$ (recall that it refers only to the fibre $\{(0,0, z, 0)\}$ ), and that of $g_{2}$ with $g_{0}$, are both evident from these definitions.

The pseudo-metric $\tilde{g}$ We can thus define the induced metric $\tilde{g}: X \rightarrow V^{*} \otimes V^{*}$ (it can be described as $g_{2} \cup\left(g_{1} \cup g_{0}\right)$, although we did not quite introduce this notation; in any case, this is not an instance of gluing of smooth maps):

$$
\tilde{g}(x, y, 0,0)= \begin{cases}\left(x^{2}+1\right) d z^{2} & \text { if } y=0, x \neq 0 \\ \left(y^{2}+1\right) d w^{2} & \text { if } x=0, y \neq 0 \\ d z^{2}+d w^{2} & \text { if } x=y=0\end{cases}
$$




\subsubsection{Relation to $T^{d v s}(\hat{X})$}

There is an obvious identification of our $X$ with $\hat{X}$, of course, and that extends to individual fibres. That is, for any $x \in X$ the fibre $\pi^{-1}(x) \subset V$ is the same as the fibre in $T^{d v s}(\hat{X})$ over the counterpart of $x$; i.e., it the standard $\mathbb{R}^{2}$ over the crossing point of the two lines, and it is $\mathbb{R}$ elsewhere. However, as we already mentioned, the diffeology of $X$ is strictly finer (smaller) than that of $\hat{X}$, and the same is true of the diffeologies of $V$ and of $T^{d v s}(\hat{X})$.

\subsection{The Clifford algebra $C \ell(V, \tilde{g})$ and gluing}

All our pseudo-bundle involve fibres of dimension at most 2, so the corresponding pseudo-bundles of Clifford algebras have dimension at most 4 . Thus, we will represent them as subsets in $\mathbb{R}^{6}$, where the first four coordinates $x, y, z, w$ correspond to the above copy of $\mathbb{R}^{4}$ that contains the pseudo-bundles themselves, the 5 -th coordinate $u_{0}$ corresponds to the scalar parts (elements of degree 0 ) of the Clifford algebras, and the 6 -th coordinate $u_{2}$ corresponds to the elements of degree 2 ; it is written with respect to the generator $\frac{\partial}{\partial z} \otimes \frac{\partial}{\partial w}$.

The Clifford algebra $C \ell\left(V_{0}, g_{0}\right)$ As a set, it is given by

$$
C \ell\left(V_{0}, g_{0}\right)=\left\{\left(0,0, z, w, u_{0}, u_{2}\right)\right\} .
$$

The Clifford multiplication, which is in fact that of the Clifford algebra of the usual $\mathbb{R}^{2}$ with the canonical scalar product, is explicitly described by:

$$
\begin{array}{r}
\left(0,0, z_{1}, w_{1}, u_{0}^{\prime}, u_{2}^{\prime}\right) \cdot C \ell\left(0,0, z_{2}, w_{2}, u_{0}^{\prime \prime}, u_{2}^{\prime \prime}\right)= \\
=\left(0,0, u_{0}^{\prime} z_{2}+u_{0}^{\prime \prime} z_{1}+2 w_{1} u_{2}^{\prime \prime}-2 u_{2}^{\prime} w_{2}, u_{0}^{\prime \prime} w_{1}+u_{0}^{\prime} w_{2}-2 z_{1} u_{2}^{\prime \prime}+2 u_{2}^{\prime} z_{2}, u_{0}^{\prime} u_{0}^{\prime \prime}-2 z_{1} z_{2}-2 w_{1} w_{2}-\right. \\
\left.u_{2}^{\prime} u_{2}^{\prime \prime}, u_{0}^{\prime} u_{2}^{\prime \prime}+u_{0}^{\prime \prime} u_{2}^{\prime}+z_{1} w_{2}-w_{1} z_{2}\right) .
\end{array}
$$

The Clifford algebras $C \ell\left(V_{1}, g_{1}\right)$ and $C \ell\left(V_{2}, g_{2}\right)$ Consistently with the above, these two are given by the sets

$$
C \ell\left(V_{1}, g_{1}\right)=\{(x, 0, z, 0, u, 0)\} \text { and } C \ell\left(V_{2}, g_{2}\right)=\{(0, y, 0, w, u, 0)\} ;
$$

the Clifford multiplication is defined, respectively, by

$$
\begin{gathered}
\left(x, 0, z_{1}, 0, u_{0}^{\prime}, 0\right) \cdot C \ell\left(x, 0, z_{2}, 0, u_{0}^{\prime \prime}, 0\right)=\left(x, 0, u_{0}^{\prime \prime} z_{1}+u_{0}^{\prime} z_{2}, 0, u_{0}^{\prime} u_{0}^{\prime \prime}-2 z_{1} z_{2}\left(x^{2}+1\right), 0\right), \\
\left(0, y, 0, w_{1}, u_{0}^{\prime}, 0\right) \cdot C \ell\left(0, y, 0, w_{2}, u_{0}^{\prime \prime}, 0\right)=\left(0, y, u_{0}^{\prime \prime} w_{1}+u_{0}^{\prime} w_{2}, 0, u_{0}^{\prime} u_{0}^{\prime \prime}-2 w_{1} w_{2}\left(y^{2}+1\right), 0\right) .
\end{gathered}
$$

The resulting Clifford algebra $C \ell(V, \tilde{g})$ It is a reflection of what has been proven in Section 5 regarding the existence of the induced gluing between (pseudo-bundles of) Clifford algebras, that we obtain a presentation of $C \ell(V, \tilde{g})$ by simply uniting the above presentations for the factors:

$$
C \ell(V, \tilde{g})=\left\{\left(0,0, z, w, u_{0}, u_{2}\right)\right\} \cup\{(x, 0, z, 0, u, 0)\} \cup\{(0, y, 0, w, u, 0)\}
$$

and

$$
\begin{aligned}
& \left(x, y, z_{1}, w_{1}, u_{0}^{\prime}, u_{2}^{\prime}\right) \cdot C \ell\left(x, y, z_{2}, w_{2}, u_{0}^{\prime \prime}, u_{2}^{\prime \prime}\right)= \\
& = \begin{cases}\left(0,0, u_{0}^{\prime} z_{2}+u_{0}^{\prime \prime} z_{1}+2 w_{1} u_{2}^{\prime \prime}-2 u_{2}^{\prime} w_{2}, u_{0}^{\prime \prime} w_{1}+u_{0}^{\prime} w_{2}-2 z_{1} u_{2}^{\prime \prime}+2 u_{2}^{\prime} z_{2},\right. & \\
\left.u_{0}^{\prime} u_{0}^{\prime \prime}-2 z_{1} z_{2}-2 w_{1} w_{2}-u_{2}^{\prime} u_{2}^{\prime \prime}, u_{0}^{\prime} u_{2}^{\prime \prime}+u_{0}^{\prime \prime} u_{2}^{\prime}+z_{1} w_{2}-w_{1} z_{2}\right) & \text { if } x=y=0, \\
\left(x, 0, u_{0}^{\prime \prime} z_{1}+u_{0}^{\prime} z_{2}, 0, u_{0}^{\prime} u_{0}^{\prime \prime}-2 z_{1} z_{2}\left(x^{2}+1\right), 0\right) & \text { if } y=0 \\
\left(0, y, u_{0}^{\prime \prime} w_{1}+u_{0}^{\prime} w_{2}, 0, u_{0}^{\prime} u_{0}^{\prime \prime}-2 w_{1} w_{2}\left(y^{2}+1\right), 0\right) & \text { if } x=0 .\end{cases}
\end{aligned}
$$

The fact that these formulae are consistent at all points of intersection reflects the commutativity of the Clifford algebra construction with gluing, established in Theorem 5.6; indeed, all the gluing maps are restrictions, to appropriate subsets, of the identity map $\mathbb{R}^{6} \rightarrow \mathbb{R}^{6}$. 


\subsection{The exterior algebras $\bigwedge V$ and $\bigwedge V_{i}$ as Clifford modules}

The four exterior algebras are defined by the same four subsets of $\mathbb{R}^{6}$; the product, being the usual exterior product, has of course different form.

The exterior algebra $\bigwedge V_{0}$ This is the set

$$
\bigwedge V_{0}=\left\{\left(0,0, z, w, u_{0}, u_{2}\right)\right\}
$$

the exterior product is given by

$\left(0,0, z_{1}, w_{1}, u_{0}^{\prime}, u_{2}^{\prime}\right) \wedge\left(0,0, z_{2}, w_{2}, u_{0}^{\prime \prime}, u_{2}^{\prime \prime}\right)=$

$$
=\left(0,0, u_{0}^{\prime} z_{2}+u_{0}^{\prime \prime} z_{1}, u_{0}^{\prime} w_{2}+u_{0}^{\prime \prime} w_{1}, u_{0}^{\prime} u_{0}^{\prime \prime}, u_{0}^{\prime} u_{2}^{\prime \prime}+u_{0}^{\prime \prime} u_{2}^{\prime}+z_{1} w_{2}-z_{2} w_{1}\right) .
$$

The exterior algebras $\bigwedge V_{1}$ and $\bigwedge V_{2}$ These are sets

$$
\bigwedge V_{1}=\{(x, 0, z, 0, u, 0)\} \text { and } \bigwedge V_{2}=\{(0, y, 0, w, u, 0)\}
$$

the respective exterior products are given by

$$
\begin{gathered}
\left(x, 0, z_{1}, 0, u_{1}, 0\right) \wedge\left(x, 0, z_{2}, 0, u_{2}, 0\right)=\left(x, 0, u_{2} z_{1}+u_{1} z_{2}, 0, u_{1} u_{2}, 0\right), \\
\left(0, y, 0, w_{1}, u_{1}, 0\right) \wedge\left(0, y, 0, w_{2}, u_{2}, 0\right)=\left(0, y, 0, u_{2} w_{1}+u_{1} w_{2}, u_{1} u_{2}, 0\right) .
\end{gathered}
$$

The exterior algebra $\bigwedge V$ Finally, the exterior algebra $\bigwedge V$ is also obtained by uniting the three presentations:

$$
\bigwedge V=\left\{\left(0,0, z, w, u_{0}, u_{2}\right)\right\} \cup\{(x, 0, z, 0, u, 0)\} \cup\{(0, y, 0, w, u, 0)\} ;
$$

the exterior product is given by

$$
\begin{aligned}
& \left(x, y, z_{1}, w_{1}, u_{0}^{\prime}, u_{2}^{\prime}\right) \wedge\left(x, y, z_{2}, w_{2}, u_{0}^{\prime \prime}, u_{2}^{\prime \prime}\right)= \\
& \quad= \begin{cases}=\left(x, y, u_{0}^{\prime} z_{2}+u_{0}^{\prime \prime} z_{1}, u_{0}^{\prime} w_{2}+u_{0}^{\prime \prime} w_{1}, u_{0}^{\prime} u_{0}^{\prime \prime}, u_{0}^{\prime} u_{2}^{\prime \prime}+u_{0}^{\prime \prime} u_{2}^{\prime}+z_{1} w_{2}-z_{2} w_{1}\right) & \text { if } x=y=0, \\
\left(x, y, u_{2} z_{1}+u_{1} z_{2}, 0, u_{1} u_{2}, 0\right) & \text { if } y=0 \\
\left(0, y, 0, u_{2} w_{1}+u_{1} w_{2}, u_{1} u_{2}, 0\right) & \text { if } x=0 .\end{cases}
\end{aligned}
$$

In fact, this is an instance of the similar commutativity of gluing with the construction of the exterior algebras' pseudo-bundles (see [10]); once again, all formulae are consistent with each other on all intersection subsets.

\subsection{The gluing of Clifford actions}

Finally, we write down the standard Clifford actions of the above Clifford algebras on the corresponding exterior algebras.

The action $c_{0}$ of $C \ell\left(V_{0}, g_{0}\right)$ on $\Lambda V_{0}$ Let us now specify, relative to our presentation of the two pseudo-bundle, the shape of the standard Clifford action of $C \ell\left(V_{0}, g_{0}\right)$ on $\bigwedge V_{0}$. To avoid too complicated formulae, and following the standard way, we specify it this time on the degree 1 generators of $C \ell\left(V_{0}, g_{0}\right)$, which are

$$
(0,0,1,0,0,0) \text { and }(0,0,0,1,0,0) \text {. }
$$

We obtain

$$
\begin{aligned}
& c_{0}(0,0,1,0,0,0)\left(\left(0,0, z, w, u_{0}, u_{2}\right)\right)=\left(0,0, u_{0},-u_{2},-z, w\right), \\
& c_{0}(0,0,0,1,0,0)\left(\left(0,0, z, w, u_{0}, u_{2}\right)\right)=\left(0,0, u_{2}, u_{0},-w,-z\right) .
\end{aligned}
$$


The actions $c_{i}$ of $C \ell\left(V_{i}, g_{i}\right)$ on $\bigwedge V_{i}$ for $i=1,2$ Analogously, it suffices to specify the action $c_{1}(x, 0,1,0,0,0)$ of the degree 1 generator of $C \ell\left(V_{1}, g_{1}\right)$ and the action $c_{2}(0, y, 0,1,0,0)$ of the degree 1 generator of $C \ell\left(V_{2}, g_{2}\right)$. We obtain

$$
\begin{aligned}
& c_{1}(x, 0,1,0,0,0)\left(\left(0,0, z, 0, u_{0}, 0\right)\right)=\left(x, 0, u_{0}, 0,-z\left(x^{2}+1\right), 0\right) . \\
& c_{2}(0, y, 0,1,0,0)\left(\left(0,0,0, w, u_{0}, 0\right)\right)=\left(0, y, 0, u_{0},-w\left(y^{2}+1\right), 0\right) .
\end{aligned}
$$

The compatibility of the two actions is reflected by the fact that these expressions coincide with those in the preceding paragraph over the point $x=y=0$.

The final action $c$ of $C \ell(V, g)$ on $\wedge V$ The action $c$ now is obtained by simply concatenating the expressions for $c_{0}, c_{1}$, and $c_{2}$.

\section{References}

[1] J.D. Christensen - E. Wu, Tangent spaces and tangent bundles for diffeological spaces, arXiv:1411.5425v1.

[2] J.D. Christensen - G. Sinnamon - E. Wu, The D-topology for diffeological spaces, arXiv.math $1302.2935 \mathrm{v} 4$.

[3] G. Hector, Géometrie et topologie des espaces difféologiques, in Analysis and Geometry in Foliated Manifolds (Santiago de Compostela, 1994), World Sci. Publishing (1995), pp. 55-80.

[4] P. Iglesias-Zemmour, Fibrations difféologiques et homotopie, Thèse de doctorat d'État, Université de Provence, Marseille, 1985.

[5] P. Iglesias-Zemmour, Diffeology, Mathematical Surveys and Monographs, 185, AMS, Providence, 2013.

[6] E. Pervova, Multilinear algebra in the context of diffeology, arXiv:1504.08186v2.

[7] E. Pervova, On the notion of scalar product for finite-dimensional diffeological vector spaces, arXiv:1507.03787v1.

[8] E. Pervova, Diffeological vector pseudo-bundles, Topology and Its Applications 202 (2016), pp. 269-300.

[9] E. Pervova, Diffeological vector pseudo-bundles, and diffeological pseudo-metrics as substitutes for Riemannian metrics, arXiv:1601.00170v1.

[10] E. Pervova, Pseudo-bundles of exterior algebras as diffeological Clifford modules, arXiv:1604.04861v1.

[11] J. Roe, Elliptic operators, topology and asymptotic methods, 2nd ed., 2001, Chapman\&Hall/CRC Research Notes in Mathematics 395.

[12] J.M. Souriau, Groups différentiels, Differential geometrical methods in mathematical physics (Proc. Conf., Aix-en-Provence/Salamanca, 1979), Lecture Notes in Mathematics, 836, Springer, (1980), pp. $91-128$.

[13] J.M. Souriau, Groups différentiels de physique mathématique, South Rhone seminar on geometry, II (Lyon, 1984), Astérisque 1985, Numéro Hors Série, pp. 341-399.

[14] J.C. VÁRILly, An introduction to noncommutative geometry, EMS Series of Lectures in Mathematics, 2006.

[15] M. Vincent, Diffeological differential geometry, Master Thesis, University of Copenhagen, 2008. 
[16] J. Watts, Diffeologies, Differential Spaces, and Symplectic Geometry, PhD Thesis, 2012, University of Toronto, Canada.

[17] E. Wu, Homological algebra for diffeological vector spaces, arXiv:1406.6717v1.

University of Pisa

Department of Mathematics

Via F. Buonarroti 1C

56127 PISA - Italy

ekaterina.pervova@unipi.it 